TRANSACTIONS OF THE

AMERICAN MATHEMATICAL SOCIETY

Volume 364, Number 7, July 2012, Pages 3781-3804

S 0002-9947(2012)05555-3

Article electronically published on February 27, 2012

\title{
PHASE-TRANSLATION GROUP ACTIONS ON STRONGLY MONOTONE SKEW-PRODUCT SEMIFLOWS
}

\author{
QIANG LIU AND YI WANG
}

\begin{abstract}
We establish a convergence property for pseudo-bounded forward orbits of strongly monotone skew-product semiflows with invariant phasetranslation group actions. The results are then applied to obtain global convergence of certain chemical reaction networks whose associated systems in reaction coordinates are monotone, as well as the dynamics of certain reactiondiffusion systems in time-recurrent structure including periodicity, almost periodicity and almost automorphy.
\end{abstract}

\section{INTRODUCTION}

Monotone dynamical systems have been widely studied because these systems provide relevant mathematical unified framework for the qualitative analysis of many important equations, including second-order parabolic equations and various classes of systems of ordinary, parabolic and functional differential equations. One may see [47, 20] for a comprehensive survey on the development of this theory.

The path-breaking work by Hirsch [18, 19] showed that trajectories in strongly monotone systems have a strong tendency to be not chaotic, i.e., almost all of their $\omega$-limit sets consist of equilibria. Now it has been well known that, for smooth strongly monotone systems, the forward orbits are generically convergent to equilibria in the continuous-time case or to cycles in the discrete-time case (see, e.g. [47, 38, 39]). Recently, nonperiodic and nonautonomous equations have been attracting more attention. A unified framework to study the nonautonomous equation is the so-called skew-product semiflow generated by the equation (see [42, 43, 44, etc.). However, in contrast to the autonomous and periodic cases, the generic convergence cannot hold (see [44) in strongly monotone skew-product semiflows, even for quasi-periodic or almost periodic cases. In such cases, the failure of generic convergence is mainly because of the multiple frequencies introduced in the base flow.

Over the past 20 years, many researchers in this field tried to impose additional conditions to obtain a more useful structure and information of the $\omega$-limit sets of the orbits. One of the popular approaches is to provide reasonable assumptions to guarantee global convergence of the orbits. Such assumptions include subhomogeneity $([23,33,46,52,56,58])$, minimal equilibria ([16, 57]), a first integral with

Received by the editors September 10, 2009 and, in revised form, January 22, 2011.

2010 Mathematics Subject Classification. Primary 37B55, 37C65, 37L15, 37N25.

The second author was the corresponding author and was partially supported by NSF of China No. 10971208 and by the Finnish Center of Excellence in Analysis and Dynamics and the FRF for the Central Universities.

(C)2012 American Mathematical Society Reverts to public domain 28 years from publication 
positive gradient (5, 24, 30, 45, 53]) and orbital stability (1, 2]), etc. Surprisingly, orbital (uniform) stability can even guarantee the convergence of all orbits for skew-product semiflows (see [25]).

An alternative interesting approach is to impose group actions on monotone dynamical systems. This idea originated from the investigation of the spatio-temporal behavior of parabolic equations and systems in which the domain and the coefficients exhibit a certain symmetry (see [8, 29]). In such a case, the semiflow commutes with the action of a topological group $G$. Typical examples of such a topological group include a rotation group $S O(N)$, a translation group, etc. Mierczyński and Poláčik [31 first investigated the symmetry structure associated with a compact connected group $G$ and showed that the $\omega$-limit sets of almost all (i.e., generic) bounded orbits are symmetric with respect to $G$, which we now call asymptotic symmetry of generic orbits. Later on, such generic asymptotic symmetry was generalized by Takác [51] to discrete-time systems with connected compact group action. Ogiwara and Matano [35, 36] also relaxed the requirements of compactness of the acting group $G$ in 31 , 51, which allowed them to discuss the monotonicity of the traveling waves for reaction-diffusion equations or systems in bistable cases. We note that the translation group is an important simple example of a noncompact connected group.

For general nonperiodic systems, one cannot expect more useful generic asymptotic symmetry information because the generic convergence property failed (see 44]). Recently, one of the present authors [55] obtained the asymptotic symmetry of uniformly stable bounded orbits for skew-product semiflows with compact connected group action. However, here we emphasize that stable bounded orbits are not the "generic" ones in skew-product semiflows anymore.

The purpose of this paper is to study the global dynamics of strongly monotone skew-product semiflows with a special phase-translation group action. More precisely, for a strongly ordered Banach space $X$ with some $v \in \operatorname{Int} X_{+}$, let $G$ be the group of phase-translations

$$
a: X \rightarrow X, a \cdot x=x+a v,
$$

by a scalar $a \in \mathbb{R}$. The action of topological group $G$ commutes with the skewproduct semiflow $\Pi: \Pi_{t}(x, g)=\left(\phi_{t}(x, g), g \cdot t\right)$ (see (2.1) $)$ as follows (also called group equivalence in [31]):

$$
\phi_{t}(a \cdot x, g)=a \cdot \phi_{t}(x, g) \text { for all } t \geq 0, a \in G, x \in X \text { and } g \in Y .
$$

We will incorporate the above two different approaches, i.e., convergence and group actions, for the monotone systems into a common framework. The connection between these two different approaches is fully established in our cases.

Via the special $G$-group action, we can introduce a skew-product semiflow $\tilde{\Pi}$ (see (3.1) ) on a codimensional-one orthogonal set of $v$ in $X$. Our main result (Theorem A) indicates that such a $G$-group action plays an essential role in determining global dynamics of the skew-product semiflow $\tilde{\Pi}$, i.e., any bounded forward orbit of $\tilde{\Pi}$ will converge. Noticing that $\tilde{\Pi}$ is not monotone in general, this result essentially enables one to obtain a global convergence property for a nonmonotone skew-product semiflow, which has interesting applications to the dynamics of several benchmark models in time-dependent chemical reaction networks (see the following paragraphs and Section 4). 
Back to the original monotone skew-product semiflow $\Pi$, one can also prove that every bounded forward orbit of $\Pi$ is convergent. However, it deserves to point out (see Remark 2.7) that the forward orbit of $\Pi$ is not necessarily bounded even if its induced forward orbit of $\tilde{\Pi}$ is bounded (the forward orbit of $\Pi$ is called pseudobounded; see Definition 2.6 and Remark 3.2). Such insight will be particularly useful when we discuss the dynamics of the time-recurrent chemical reaction networks in Section 4.

In Section 4, we focus on investigating the dynamical behavior of certain classes of chemical reaction networks. In much of the earlier studies, many researchers restricted their consideration to the time-independent chemical reaction networks (see, e.g., 3, 4, 27, 41, 48, 26] and the references therein). However, in practical laboratory experiments, the system evolves influenced by external time-dependent effects which are periodic, roughly periodic, or under environmental forcing which exhibits different, noncommensurate periods. Then it is unlikely one can maintain the time-independent restriction, and it is therefore of considerable interest to study the problem when the sort of time dependence is involved. Our main results will be applied to obtain global dynamics for such chemical reaction networks with time-recurrent structure including periodicity, almost periodicity and almost automorphy. The key idea of our approach is to lift such a chemical reaction network to an alternative representation under which the resulting system (called the associated system in reaction coordinates [3, 37]) is strongly monotone. There are large quantities of models taken from the current biochemical literature admitting the monotonicity of the new system description in reaction coordinates. The benchmark examples include the phosphorylation/dephosphorylation processes (sometimes called enzyme futile cycles; see, e.g., [12, 26, 41, 4]), nonmass action kinetics under the QSSA assumption in dimerization reactions of proteins (see [3, 26]) and more complex reaction networks which arise in many signal transduction pathways, the MAPK cascade and the RKIP inhibited ERK pathway from Cho et al. [9, 3. Among them, we will choose a simple phosphorylation/dephosphorylation process in mass action kinetics as an illustrated example to show the technical detail of verifying the monotonicity for the new system in reaction coordinates.

Although the new system in reaction coordinates has been known to be strongly monotone, a careful examination immediately yields that the change to such a new system does not seem particularly useful because there is no guarantee that the solutions of the new system are bounded. (Recently, Hu and Jiang 21, 22 discussed such a new monotone system under the assumption of boundedness for every solution.) In fact, as pointed out by Angeli et al. in [3, p. 596]: "this issue constitutes the main technical difficulty that needs to be surmounted in order for us to obtain the convergence results for the system". To overcome such difficulty, motivated by [4, we therefore introduce the "pseudo-boundedness" (see Definition 2.6 and Remark 2.7) and accomplish showing that every orbit of the new system is pseudo-bounded, which enables us to obtain convergence results for the original chemical reaction networks.

Finally, we will also use our main results in Section 5 to obtain a convergence property for a certain class of time-recurrent reaction-diffusion systems.

This paper is organized as follows. In Section 2 we agree on some notation and give relevant definitions and preliminary results which will be important to our proofs. We state our main results and give their proofs in Section 3. Sections 4 and 
5 are devoted to the study of global convergence results in time-dependent chemical reaction networks and nonlinear reaction-diffusion equations for which our abstract theorems in Section 3 apply.

\section{Preliminaries}

In this section, we collect some preliminary materials that will be used later. First, we recall the definitions of partial order and the induced topology. We then summarize some definitions and basic properties of strongly monotone skew-product semiflows. Finally, we give a brief review about almost periodic functions.

Let $Y$ be a compact metric space with metric $d_{Y}$ and let $\sigma: Y \times \mathbb{R} \rightarrow Y,(g, t) \mapsto$ $g \cdot t$ be a continuous flow on $Y$, denoted by $(Y, \sigma)$ or $(Y, \mathbb{R})$. A subset $S \subset Y$ is invariant if $\sigma_{t}(S)=S$ for every $t \in \mathbb{R}$. A nonempty compact invariant set $S \subset Y$ is called minimal if it contains no nonempty, proper and invariant subset. We say that the continuous flow $(Y, \mathbb{R})$ is minimal if $Y$ itself is a minimal set. Let $(Z, \mathbb{R})$ be another continuous flow. A continuous map $p: Z \rightarrow Y$ is called a flow homomorphism if $p(z \cdot t)=p(z) \cdot t$ for all $z \in Z$ and $t \in \mathbb{R}$. Moreover, $p$ is called $a$ flow isomorphism if it is a homeomorphism from $Z$ to $Y$.

We say that $(X,\|\cdot\|)$ is a strongly ordered Banach space if there is a closed convex cone, that is, a nonempty closed subset $X_{+} \subset X$ satisfying $X_{+}+X_{+} \subset X_{+}$, $X_{+} \subset \alpha X_{+}$for all $\alpha \geq 0$, and $X_{+} \cap\left(-X_{+}\right)=\{0\}$ with nonempty interior Int $X_{+} \neq \emptyset$ (also say that $X_{+}$is solid). The cone $X_{+}$induces a strong ordering on $X$ via $x_{1} \leq x_{2}$ if $x_{2}-x_{1} \in X_{+}$. We write $x_{1}<x_{2}$ if $x_{2}-x_{1} \in X_{+} \backslash\{0\}$, and $x_{1} \ll x_{2}$ if $x_{2}-x_{1} \in \operatorname{Int} X_{+}$. Given $x_{1}, x_{2} \in X$, the set $\left[x_{1}, x_{2}\right]=\left\{x \in X: x_{1} \leq x \leq x_{2}\right\}$ is called a closed order interval in $X$ and $\left[\left[x_{1}, x_{2}\right]\right]=\left\{x \in X: x_{1} \ll x \ll x_{2}\right\}$ is called an open order interval in $X$. The cone $X_{+}$is said to be normal if the norm $\|\cdot\|$ is semimonotone, i.e., there is a constant $c$ such that the property $0 \leq x_{1} \leq x_{2}$ implies that $\left\|x_{1}\right\| \leq c\left\|x_{2}\right\|$. Define the order topology on $X$ which is induced by the ordered norm defined by $\|x\|_{e}=\inf \{\lambda>0: x \in \lambda[[-e, e]]\}$ for some $e \in \operatorname{Int} X_{+}$. In general, $\|x\|$ is stronger than $\|x\|_{e}$. If $X_{+}$is solid and normal, then the induced order topology is equivalent to the original topology (see [10, p. 230).

Throughout this paper, we always assume that the flow $(Y, \mathbb{R})$ is minimal and $X$ is a strongly ordered Banach space with normal cone $X_{+}$.

Let $\mathbb{R}^{+}=\{t \in \mathbb{R}: t \geq 0\}$. We consider a continuous skew-product semiflow $\Pi: X \times Y \times \mathbb{R}^{+} \rightarrow X \times Y$ defined by

$$
\Pi_{t}(x, g)=\left(\phi_{t}(x, g), g \cdot t\right), \quad \forall(x, g, t) \in X \times Y \times \mathbb{R}^{+},
$$

satisfying (1) $\Pi_{0}=\mathrm{Id} ;(2)$ the cocycle property $\phi_{t+s}(x, g)=\phi_{s}\left(\phi_{t}(x, g), g \cdot t\right)$ for each $(x, g) \in X \times Y$ and $s, t \in \mathbb{R}^{+}$.

A subset $A \subset X \times Y$ is positively invariant if $\Pi_{t}(A) \subset A$ for all $t \in \mathbb{R}^{+}$and is totally invariant if $\Pi_{t}(A)=A$ for all $t \in \mathbb{R}^{+}$. The forward orbit of any $(x, g) \in X \times Y$ is defined by $\mathcal{O}^{+}(x, g)=\left\{\Pi_{t}(x, g): t \geq 0\right\}$, and the $\omega$-limit set of $(x, g)$ is defined by $\omega(x, g)=\left\{(\hat{x}, \hat{g}) \in X \times Y: \Pi_{t_{n}}(x, g) \rightarrow(\hat{x}, \hat{g})(n \rightarrow \infty)\right.$ for some sequence $t_{n} \rightarrow$ $\infty$ \}. A skew-product semiflow $\left(X \times Y, \Pi, \mathbb{R}^{+}\right)$is called completely continuous if for any bounded set $E \subset X, \Pi_{t}(E \times Y)$ is relatively compact for any $t>0$. Clearly, if $\Pi$ is completely continuous, then the omega-limit set $\mathcal{O}(\omega, x)$ of every bounded forward orbit $\mathcal{O}^{+}(x, g)$ is a nonempty, compact and totally invariant subset in $\Omega \times X$ for $\Pi$. 
A flow extension of $\left(X \times Y, \Pi, \mathbb{R}^{+}\right)$is a continuous skew-product flow $(X \times Y, \hat{\Pi}, \mathbb{R})$ such that $\Pi(x, g, t)=\hat{\Pi}(x, g, t)$ for each $(x, g) \in X \times Y$ and all $t \in \mathbb{R}^{+}$. A compact positively invariant subset is said to admit a flow extension if the semiflow restricted to it does as well. Actually, a compact positively invariant set $A \subset X \times Y$ admits a flow extension if every point in $A$ admits a unique backward orbit which remains inside the set $A$ (see [4]).

Assume that $E \subset X \times Y$ is a compact positively invariant set for $\Pi$ which admits a flow extension. Let $p: X \times Y \rightarrow Y$ be the natural projection. Then $p$ is a flow homomorphism for the flows $(E, \mathbb{R})$ and $(Y, \sigma)$.

A set $E \subset X \times Y$ is said to be positively fiber distal if for any $g \in Y$,

$$
\inf _{t \in \mathbb{R}^{+}}\left\|\phi_{t}\left(x_{1}, g\right)-\phi_{t}\left(x_{2}, g\right)\right\|>0
$$

whenever $\left(x_{i}, g\right) \in E \cap p^{-1}(g)$ for $i=1,2$. A compact invariant subset $E \subset X \times Y$ of $\Pi$ is called a 1-cover of $Y$ based on $\Pi$ if $p^{-1}(g) \cap E$ is a singleton for any $g \in Y$.

The strong ordering on $X$ induces a strong ordering on $X \times Y$ as follows:

$$
\begin{gathered}
\left(x_{1}, g\right) \leq\left(x_{2}, g\right) \quad \Leftrightarrow \quad x_{1} \leq x_{2}, \\
\left(x_{1}, g\right)<\left(x_{2}, g\right) \Leftrightarrow x_{1}<x_{2}, \\
\left(x_{1}, g\right) \ll\left(x_{2}, g\right) \Leftrightarrow x_{1} \ll x_{2} .
\end{gathered}
$$

In other words, for skew-product semiflows, we use the order relation on each fiber $p^{-1}(g)$. We write $\left(x_{1}, g\right) \leq_{g}\left(<_{g}, \ll_{g}\right)\left(x_{2}, g\right)$ if $x_{1} \leq x_{2}\left(x_{1}<x_{2}, x_{1} \ll x_{2}\right)$. Without any confusion, we will drop the subscript " $g$ ". One can also define similar definitions and notation in $p^{-1}(g)$ as in $X$, such as order-intervals, etc.

Definition 2.1. The skew-product semiflow $\Pi$ is monotone if

$$
\Pi_{t}\left(x_{1}, g\right) \leq \Pi_{t}\left(x_{2}, g\right)
$$

whenever $\left(x_{1}, g\right) \leq\left(x_{2}, g\right)$ and $t \geq 0$. Moreover, $\Pi$ is strongly monotone if it is monotone and

$$
\Pi_{t}\left(x_{1}, g\right) \ll \Pi_{t}\left(x_{2}, g\right) \text { whenever }\left(x_{1}, g\right)<\left(x_{2}, g\right) \text { and } t>0 .
$$

Definition 2.2 (Uniform stability). A forward orbit $\mathcal{O}^{+}\left(x_{0}, g_{0}\right)$ of $\Pi$ is said to be uniformly stable if for every $\epsilon>0$ there is a $\delta=\delta(\epsilon)>0$ such that if $s \geq 0$ and $\left\|x-\phi_{s}\left(x_{0}, g_{0}\right)\right\| \leq \delta(\epsilon)$ for certain $x \in X$, then for each $t \geq 0$,

$$
\left\|\phi_{t}\left(x, g_{0} \cdot s\right)-\phi_{s+t}\left(x_{0}, g_{0}\right)\right\|=\left\|\phi_{t}\left(x, g_{0} \cdot s\right)-\phi_{t}\left(\phi_{s}\left(x_{0}, g_{0}\right), g_{0} \cdot s\right)\right\| \leq \epsilon \text {. }
$$

The following two results are adopted from [34, 44] and will play important roles in our forthcoming sections.

Lemma 2.3. Assume that a forward orbit $\mathcal{O}^{+}(x, g)$ is relatively compact and uniformly stable. Then the $\omega$-limit set $\omega(x, g)$ is a minimal set which admits a fiber distal flow extension.

Proof. It follows from Theorem 3.4 in [34].

Lemma 2.4. Assume that $\Pi$ is strongly monotone and let $K$ be a minimal set of $X \times Y$ which admits a flow extension. If $K$ is fiber distal, then no two points on the same fiber are ordered.

Proof. It follows from Theorem 2.3.2 and Corollary 2.3.3 in [44]. 
Fix $v \in \operatorname{Int} X_{+}$with $\|v\|=1$. Let $G$ be the group of phase-translation

$$
a: X \rightarrow X ; a \cdot x=x+a v,
$$

by a scalar $a \in \mathbb{R}$.

Definition 2.5. The phase-translation group $G$ commutes with the skew-product semiflow $\Pi$ if

$$
\phi_{t}(a \cdot x, g)=a \cdot \phi_{t}(x, g)
$$

for any $(x, y) \in X \times Y, t \geq 0$ and $a \in G$.

For such $v$ above, the Banach space $X$ has a direct sum decomposition

$$
X=X_{0} \oplus \operatorname{span}\{v\},
$$

where $X_{0}$ is the null space of a bounded linear functional $f$ on $X$ with $\langle f, v\rangle=1$. A natural projection on $X_{0}$ is defined as

$$
\pi: X \rightarrow X_{0}: x \mapsto x-\langle f, x\rangle v .
$$

Definition 2.6. A forward orbit $\mathcal{O}^{+}(x, g)$ of $\Pi$ is said to be pseudo-bounded if $\left(\pi \phi_{t}(x, g), g \cdot t\right)$ is bounded in $X \times Y$ for all $t \geq 0$.

Remark 2.7. Clearly, "Boundedness" $\Longrightarrow$ "Pseudo-boundedness". However, the reverse is not true. As a simple counterexample, we consider the following autonomous system of ODEs:

$$
\left\{\begin{array}{l}
\dot{x}=-x+y+1, \quad t>0 . \\
\dot{y}=x-y+1,
\end{array}\right.
$$

A direct calculation yields the solution, with the initial value $u_{0}=\left(x_{0}, y_{0}\right)^{T}$,

$$
\phi_{t}\left(u_{0}\right)=\left(\begin{array}{c}
x\left(t ; u_{0}\right) \\
y\left(t ; u_{0}\right)
\end{array}\right)=\left(\begin{array}{c}
t+\frac{1}{2}\left(x_{0}+y_{0}\right)+\frac{1}{2}\left(x_{0}-y_{0}\right) e^{-2 t} \\
t+\frac{1}{2}\left(x_{0}+y_{0}\right)-\frac{1}{2}\left(x_{0}-y_{0}\right) e^{-2 t}
\end{array}\right) .
$$

Choose $v=\left(\frac{1}{2}, \frac{1}{2}\right)^{T} \in \operatorname{Int} \mathbb{R}_{+}^{2}$ and let $G$ be the phase-translation group w.r.t. $v$. Then $G$ commutes with the flow of (2.5) $)$. It is easy to see that every solution $\phi_{t}\left(u_{0}\right)$ is pseudo-bounded (with $X_{0}=\left\{(x, y) \in \mathbb{R}^{2}: x+y=0\right\}$ ), but not bounded.

We finish this section with the definitions of almost periodic (almost automorphic) functions and flows.

A function $f \in C\left(\mathbb{R}, \mathbb{R}^{n}\right)$ is almost periodic if, for any $\varepsilon>0$, the set $T(\varepsilon):=\{\tau$ : $|f(t+\tau)-f(t)|<\varepsilon, \forall t \in \mathbb{R}\}$ is relatively dense in $\mathbb{R}$. $f$ is almost automorphic if for any $\left\{t_{n}^{\prime}\right\} \subset \mathbb{R}$ there is a subsequence $\left\{t_{n}\right\}$ and a function $g: \mathbb{R} \rightarrow \mathbb{R}^{n}$ such that $f\left(t+t_{n}\right) \rightarrow g(t)$ and $g\left(t-t_{n}\right) \rightarrow f(t)$ hold pointwise.

Let $D \subseteq \mathbb{R}^{m}$ be a subset of $\mathbb{R}^{m}$. A continuous function $f: \mathbb{R} \times D \rightarrow \mathbb{R}^{n} ;(t, u) \mapsto$ $f(t, u)$ is said to be admissible if $f(t, u)$ is bounded and uniformly continuous on $\mathbb{R} \times K$ for any compact subset $K \subset D$. A function $f \in C\left(\mathbb{R} \times D, \mathbb{R}^{n}\right)\left(D \subset \mathbb{R}^{m}\right)$ is uniformly almost periodic (almost automorphic) in $t$ if $f$ is both admissible and almost periodic (almost automorphic) in $t \in \mathbb{R}$.

Let $f \in C\left(\mathbb{R} \times D, \mathbb{R}^{n}\right)\left(D \subset \mathbb{R}^{m}\right)$ be admissible. Then $H(f)=\operatorname{cl}\{f \cdot \tau: \tau \in \mathbb{R}\}$ is called the hull of $f$, where $f \cdot \tau(t, \cdot)=f(t+\tau, \cdot)$ and the closure is taken under the compact open topology. Moreover, $H(f)$ is compact and metrizable under the compact open topology (see [43, 44]). The time translation $g \cdot t$ of $g \in H(f)$ induces a natural flow on $H(f)$ (cf. [43]). 
Definition 2.8. An admissible function $f \in C\left(\mathbb{R} \times D, \mathbb{R}^{n}\right)\left(D \subset \mathbb{R}^{m}\right)$ is called time-recurrent if $H(f)$ is minimal.

Remark 2.9. $H(f)$ is always minimal if $f$ is periodic or uniformly almost periodic (almost automorphic) in $t$ (see, e.g. [4]).

Let $f \in C\left(\mathbb{R} \times \mathbb{R}^{n}, \mathbb{R}^{n}\right)$ be a uniformly almost periodic (almost automorphic) function, and let

$$
f(t, x) \sim \sum_{\lambda \in \mathbb{R}} a_{\lambda}(x) e^{i \lambda t}
$$

be a Fourier series of $f$ (see [54, 45] for the definition and the existence of a Fourier series). Then $\mathcal{S}=\left\{\lambda: a_{\lambda}(x) \not \equiv 0\right\}$ is called the Fourier spectrum of $f$ associated to the Fourier series (2.6), and $\mathcal{M}(f)=$ the smallest additive subgroup of $\mathbb{R}$ containing $\mathcal{S}(f)$ is called the frequency module of $f$. Moreover, $\mathcal{M}(f)$ is a countable subset of $\mathbb{R}$. Let $f, g \in C\left(\mathbb{R} \times \mathbb{R}^{n}, \mathbb{R}^{n}\right)$ be two uniformly almost periodic (almost automorphic) functions in $t$. The module containment $\mathcal{M}(f) \subseteq \mathcal{M}(g)$ if and only if there exists a flow epimorphism from $H(g)$ to $H(f)$ (see [14] or [44, Section 1.3.4]).

\section{MAin RESUlts AND PROOFS}

In this section, we first collect our standing assumptions as follows:

(A1) $Y$ is minimal, $X$ is a strongly ordered Banach space with normal cone $X_{+}$;

(A2) the skew-product semiflow $\Pi$ on $X \times Y$ is strongly monotone and completely continuous;

(A3) the phase-translation group $G$ commutes with $\Pi$ (see Definition 2.5).

We introduce an induced continuous mapping $\tilde{\Pi}$ by $\Pi_{t}, t \geq 0$, as follows:

$$
\begin{aligned}
& \tilde{\Pi}_{t}: X_{0} \times Y \rightarrow X_{0} \times Y, \\
& (\tilde{x}, g) \quad \mapsto \quad\left(\tilde{\phi}_{t}(\tilde{x}, g), g \cdot t\right):=\left(\pi \phi_{t}(\tilde{x}, g), g \cdot t\right),
\end{aligned}
$$

where $X_{0}, \pi$ are defined in (2.3) and (2.4). Then $\tilde{\Pi}$ satisfies the following:

Proposition 3.1. The mapping $\tilde{\Pi}$ is a skew-product semiflow on $X_{0} \times Y$. Moreover, for any $(x, g) \in X \times Y$ and $t \geq 0$, one has

$$
\pi \phi_{t}(x, g)=\tilde{\phi}_{t}(\pi x, g) \text {. }
$$

Proof. We first prove (3.2). For any $(x, g) \in X \times Y$ and $t \geq 0$, by the definition of $\tilde{\Pi}$ one has $\tilde{\phi}_{t}(\pi x, g)=\pi \phi_{t}(\pi x, g)$. Moreover, it follows from (A3) and the definition of the projection $\pi$ that

$$
\pi \phi_{t}(x, g)=\pi \phi_{t}(\pi x+\langle f, x\rangle v, g)=\pi\left(\phi_{t}(\pi x, g)+\langle f, x\rangle v\right)=\pi \phi_{t}(\pi x, g) .
$$

As a consequence, $\pi \phi_{t}(x, g)=\tilde{\phi}_{t}(\pi x, g)$.

For any $(\tilde{x}, g) \in X_{0} \times Y$, pick an $x \in \pi^{-1}(\tilde{x})$. By virtue of (3.2), we have $\tilde{\phi}_{0}(\tilde{x}, g)=\pi \phi_{0}(x, g)=\pi x=\tilde{x}$. Moreover, for every $t, s \geq 0$,

$$
\begin{aligned}
\tilde{\phi}_{s+t}(\tilde{x}, g) & =\pi \phi_{s+t}(x, g)=\pi \phi_{s}\left(\phi_{t}(x, g), g \cdot t\right) \\
& =\tilde{\phi}_{s}\left(\pi \phi_{t}(x, g), g \cdot t\right)=\tilde{\phi}_{s}\left(\tilde{\phi}_{t}(\tilde{x}, g), g \cdot t\right) .
\end{aligned}
$$

Thus, we proved that $\tilde{\Pi}$ is a skew-product semiflow on $X_{0} \times Y$.

From now on, for any $(\tilde{x}, g) \in X_{0} \times Y$, we denote by $\tilde{\mathcal{O}}^{+}(\tilde{x}, g)$ and $\tilde{\omega}(\tilde{x}, g)$ the forward orbit and the omega-limit set with respect to $\tilde{\Pi}$, respectively. 
Remark 3.2. The pseudo-boundedness of an orbit $\mathcal{O}^{+}(x, g)$ implies the boundedness of the induced orbit $\tilde{\mathcal{O}}^{+}(\pi x, g)$. On the contrary, if $\tilde{\mathcal{O}}^{+}(\tilde{x}, g)$ is bounded, then $\mathcal{O}^{+}(x, g)$ is pseudo-bounded for any $x \in \pi^{-1}(\tilde{x})$.

Now we present our main results.

Theorem A. Assume that $X, Y$ and $\Pi$ satisfy (A1)-(A3). Let $\left(\tilde{x}_{1}, g\right),\left(\tilde{x}_{2}, g\right) \in$ $X_{0} \times Y$ be such that their orbits $\tilde{\mathcal{O}}^{+}\left(\tilde{x}_{1}, g\right)$ and $\tilde{\mathcal{O}}^{+}\left(\tilde{x}_{2}, g\right)$ are bounded. Then it holds that

$$
\left\|\tilde{\phi}_{t}\left(\tilde{x}_{1}, g\right)-\tilde{\phi}_{t}\left(\tilde{x}_{2}, g\right)\right\| \rightarrow 0 \quad \text { as } t \rightarrow \infty .
$$

In particular, the following statements hold:

(i) There exists at most one 1-cover of $Y$ with respect to $\tilde{\Pi}$.

(ii) For any bounded orbit $\tilde{\mathcal{O}}^{+}(\tilde{x}, g)$, the omega-limit set $\tilde{\omega}(\tilde{x}, g)$ is a 1-cover of $Y$ with respect to $\tilde{\Pi}$.

Theorem B. Assume that $X, Y$ and $\Pi$ satisfy (A1)-(A3). Let $\mathcal{O}^{+}(x, g)$ be a forward orbit of $\Pi$. Then

1) the omega-limit set $\omega(x, g)$ of $\mathcal{O}^{+}(x, g)$ is a 1-cover of $Y$ if $\mathcal{O}^{+}(x, g)$ is bounded;

2) the omega-limit set $\tilde{\omega}(\pi x, g)$ of $\tilde{\mathcal{O}}^{+}(\pi x, g)$ is a 1-cover of $Y$ if $\mathcal{O}^{+}(x, g)$ is pseudo-bounded.

Before addressing the technical steps of the proof, it is helpful to build up some useful lemmas.

Lemma 3.3. For any $\left(x_{0}, g_{0}\right) \in X \times Y$, the forward orbit $\mathcal{O}^{+}\left(x_{0}, g_{0}\right)$ of $\Pi$ is uniformly stable.

Proof. Since $X$ is normal, it suffices to show that $\mathcal{O}^{+}\left(x_{0}, g_{0}\right)$ is uniformly stable with respect to $\|\cdot\|_{v}$. For any $\varepsilon>0$, choose $\delta=\varepsilon$. For every $x \in X$, if $s \geq 0$ and $\left\|x-\phi_{s}\left(x_{0}, g_{0}\right)\right\|_{v} \leq \delta$, we have $\phi_{s}\left(x_{0}, g_{0}\right)-\delta v \ll x \ll \phi_{s}\left(x_{0}, g_{0}\right)+\delta v$. It then follows from monotonicity of $\Pi$ that

$$
\phi_{t}\left(\phi_{s}\left(x_{0}, g_{0}\right)-\delta v, g_{0} \cdot s\right) \ll \phi_{t}\left(x, g_{0} \cdot s\right) \ll \phi_{t}\left(\phi_{s}\left(x_{0}, g_{0}\right)+\delta v, g_{0} \cdot s\right),
$$

for each $t \geq 0$. By virtue of (A3), one obtains

$$
\phi_{t+s}\left(x_{0}, g_{0}\right)-\delta v \ll \phi_{t}\left(x, g_{0} \cdot s\right) \ll \phi_{t+s}\left(x_{0}, g_{0}\right)+\delta v,
$$

and hence $\left\|\phi_{t}\left(x, g_{0} \cdot s\right)-\phi_{t+s}\left(x_{0}, g_{0}\right)\right\|_{v} \leq \delta=\varepsilon$.

Remark 3.4. By appealing to the results in [25], Lemma 3.3 may imply the global convergence provided that EACH orbit of $\Pi$ is bounded (and hence relatively compact since $\Pi$ is completely continuous). In our case, however, $\Pi$ may possess pseudobounded rather than bounded orbits (see Remark 2.7). Consequently, we are led to consider the induced nonmonotone skew-product semiflow $\tilde{\Pi}$ in (3.1) independently:

Lemma 3.5. For every $\left(\tilde{x}_{0}, g_{0}\right) \in X_{0} \times Y$, the induced forward orbit $\tilde{\mathcal{O}}^{+}\left(\tilde{x}_{0}, g_{0}\right)$ is uniformly stable.

Proof. Since the projection $\pi$ is a linear bounded operator, we may assume that $\|\pi\| \leq M$ for some $M>0$. Fix an $x_{0} \in \pi^{-1}\left(\tilde{x}_{0}\right)$. For any $\varepsilon>0$, it follows from 
Lemma 3.3 that there exists some $\delta(\varepsilon)>0$ such that

$$
\left\|\phi_{t}\left(x, g_{0} \cdot s\right)-\phi_{s+t}\left(x_{0}, g_{0}\right)\right\|<\varepsilon / M \quad(t \geq 0)
$$

whenever $s \geq 0, x \in X$ and $\left\|x-\phi_{s}\left(x_{0}, g_{0}\right)\right\|<\delta$.

Given any $\tilde{x} \in X_{0}$ and $s \geq 0$ with $\left\|\tilde{x}-\tilde{\phi}_{s}\left(\tilde{x}_{0}, g_{0}\right)\right\|<\delta$, by virtue of Proposition 3.1. we obtain that

$$
\left\|\pi x-\pi \phi_{s}\left(x_{0}, g_{0}\right)\right\|=\left\|\tilde{x}-\tilde{\phi}_{s}\left(\tilde{x}_{0}, g_{0}\right)\right\|<\delta,
$$

whenever $x \in \pi^{-1}(\tilde{x})$.

Choose an $x \in \pi^{-1}(\tilde{x})$. Note that

$$
x-\phi_{s}\left(x_{0}, g_{0}\right)=\pi x-\pi \phi_{s}\left(x_{0}, g_{0}\right)-\lambda_{0} v,
$$

for some $\lambda_{0} \in \mathbb{R}$. Then, by (3.5), one has $\left\|x+\lambda_{0} v-\phi_{s}\left(x_{0}, g_{0}\right)\right\|<\delta$. Together with (3.4), we deduce that $\left\|\phi_{t}\left(x+\lambda_{0} v, g_{0} \cdot s\right)-\phi_{s+t}\left(x_{0}, g_{0}\right)\right\|<\varepsilon / M$, for all $t \geq 0$. Thus, $\left\|\pi \phi_{t}\left(x+\lambda_{0} v, g_{0} \cdot s\right)-\pi \phi_{s+t}\left(x_{0}, g_{0}\right)\right\|<\varepsilon$, for all $t \geq 0$. Again by Proposition 3.1, we obtain that

$$
\left\|\tilde{\phi}_{t}\left(\tilde{x}, g_{0} \cdot s\right)-\tilde{\phi}_{s+t}\left(\tilde{x}_{0}, g_{0}\right)\right\| \leq \varepsilon \quad \text { for all } t \geq 0 .
$$

This completes the proof.

Lemma 3.6. If an orbit $\mathcal{O}^{+}(x, g)$ is pseudo-bounded, then its induced orbit $\tilde{\mathcal{O}}^{+}(\pi x, g)$ is relatively compact in $X_{0} \times Y$.

Proof. By Remark 3.2, the induced orbit $\tilde{\mathcal{O}}^{+}(\pi x, g)$ is bounded in $X_{0} \times Y$. Since $\Pi$ is completely continuous, $\Pi_{\tau}\left(\tilde{\mathcal{O}}^{+}(\pi x, g)\right)$ is relatively compact for every $\tau>$ 0 , and hence $\tilde{\Pi}_{\tau}\left(\tilde{\mathcal{O}}^{+}(\pi x, g)\right)$ is relatively compact. Note that $\tilde{\Pi}_{\tau}\left(\tilde{\mathcal{O}}^{+}(\pi x, g)\right)=$ $\left\{\tilde{\Pi}_{t}(\pi x, g): t \geq \tau\right\}$. It then follows that $\left\{\tilde{\Pi}_{t}(\pi x, g): t \geq \tau\right\}$ is relatively compact for any fixed $\tau>0$. Thus, $\tilde{\mathcal{O}}^{+}(\pi x, g)$ is relatively compact in $X_{0} \times Y$.

Together with Lemmas 3.5 and 3.6, we have the following

Lemma 3.7. Suppose that the orbit $\mathcal{O}^{+}(x, g)$ is pseudo-bounded. Then the omegalimit set $\tilde{\omega}(\pi x, g)$ of $\tilde{\Pi}$ is minimal and admits a fiber distal flow extension.

Proof. It follows from Lemma 2.3. Lemma 3.5 and Lemma 3.6.

Now we are in a position to introduce a function $V: X \times Y \rightarrow \mathbb{R}^{+}$,

$$
V(x, g):=\inf \{\alpha \geq 0:(-\alpha v, g) \leq(x, g) \leq(\alpha v, g)\} .
$$

Lemma 3.8. Let $V$ be defined as above. Then

(i) $V$ is a well-defined nonnegative function which is continuous on $X \times Y$ and Lipschitz in $x \in X$;

$$
V\left(\phi_{t}\left(x_{1}, g\right)-\phi_{t}\left(x_{2}, g\right), g \cdot t\right) \leq V\left(x_{1}-x_{2}, g\right)
$$

for all $x_{1}, x_{2} \in X, g \in Y$ and $t>0$. Moreover, the equality in (3.6) holds if and only if $x_{1}-x_{2} \in \operatorname{span}\{v\}$.

Proof. (i) Since $[[-v, v]]$ is an open neighborhood of zero, one has $x / \alpha \in[[-v, v]]$ for all $\alpha>0$ sufficiently large. Consequently, $V(x, g)$ is a well-defined nonnegative function. It is also easy to see that $V$ is continuous on $X \times Y$. 
We now prove that $V$ is Lipschitz in $x \in X$. To end this, choose an $\varepsilon_{0}>0$ such that $(-v, g) \leq\left(\epsilon_{0} z, g\right) \leq(v, g)$ for all $\|z\|=1$. It then follows that

$$
\left(-\epsilon_{0}^{-1}\left\|x_{1}-x_{2}\right\| v, g\right) \leq\left(x_{1}-x_{2}, g\right) \leq\left(\epsilon_{0}^{-1}\left\|x_{1}-x_{2}\right\| v, g\right)
$$

for any two points $x_{1}, x_{2} \in X$. Meanwhile, it follows from the definition of $V$ that

$$
\left(-V\left(x_{2}, g\right) v, g\right) \leq\left(x_{2}, g\right) \leq\left(V\left(x_{2}, g\right) v, g\right) .
$$

By (3.7) and (3.8), one has

$$
\left(-\epsilon_{0}^{-1}\left\|x_{1}-x_{2}\right\| v-V\left(x_{2}, g\right) v, g\right) \leq\left(x_{1}, g\right) \leq\left(\epsilon_{0}^{-1}\left\|x_{1}-x_{2}\right\| v+V\left(x_{2}, g\right) v, g\right),
$$

which implies that $V\left(x_{1}, g\right) \leq \epsilon_{0}^{-1}\left\|x_{1}-x_{2}\right\|+V\left(x_{2}, g\right)$, and hence $V\left(x_{1}, g\right)-$ $V\left(x_{2}, g\right) \leq \epsilon_{0}^{-1}\left\|x_{1}-x_{2}\right\|$. Note that $x_{1}$ and $x_{2}$ are arbitrary; this implies that $\left|V\left(x_{1}, g\right)-V\left(x_{2}, g\right)\right| \leq \epsilon_{0}^{-1}\left\|x_{1}-x_{2}\right\|$. Therefore, $V$ is Lipschitz in $x \in X$.

(ii) For any $x_{1}, x_{2} \in X$ and each $g \in Y$, one has

$$
\left(x_{2}-V\left(x_{1}-x_{2}, g\right) v, g\right) \leq\left(x_{1}, g\right) \leq\left(x_{2}+V\left(x_{1}-x_{2}, g\right) v, g\right) .
$$

By virtue of monotonicity and (A3), it follows that

$$
\phi_{t}\left(x_{2}, g\right)-V\left(x_{1}-x_{2}, g\right) v \leq \phi_{t}\left(x_{1}, g\right) \leq \phi_{t}\left(x_{2}, g\right)+V\left(x_{1}-x_{2}, g\right) v,
$$

for $t \geq 0$. This implies that

(3.9) $\left(-V\left(x_{1}-x_{2}, g\right) v, g \cdot t\right) \leq\left(\phi_{t}\left(x_{1}, g\right)-\phi_{t}\left(x_{2}, g\right), g \cdot t\right) \leq\left(V\left(x_{1}-x_{2}, g\right) v, g \cdot t\right)$.

Hence $V\left(\phi_{t}\left(x_{1}, g\right)-\phi_{t}\left(x_{2}, g\right), g \cdot t\right) \leq V\left(x_{1}-x_{2}, g\right)$ for all $t \geq 0$.

Now if $x_{1}-x_{2} \in \operatorname{span}\{v\}$, then it is easy to see that

$$
V\left(\phi_{t}\left(x_{1}, g\right)-\phi_{t}\left(x_{2}, g\right), g \cdot t\right)=V\left(x_{1}-x_{2}, g\right) \text { for all } t \geq 0 .
$$

Suppose that $x_{1}-x_{2} \notin \operatorname{span}\{v\}$. Then

$$
\left(x_{2}-V\left(x_{1}-x_{2}, g\right) v, g\right)<\left(x_{1}, g\right)<\left(x_{2}+V\left(x_{1}-x_{2}, g\right) v, g\right) .
$$

By exploiting strong monotonicity of $\Pi$, one immediately obtains

$$
\phi_{t}\left(x_{2}-V\left(x_{1}-x_{2}, g\right) v, g\right) \ll \phi_{t}\left(x_{1}, g\right) \ll \phi_{t}\left(x_{2}+V\left(x_{1}-x_{2}, g\right) v, g\right) .
$$

Again by (A3), we have $-V\left(x_{1}-x_{2}, g\right) v \ll \phi_{t}\left(x_{1}, g\right)-\phi_{t}\left(x_{2}, g\right) \ll V\left(x_{1}-x_{2}, g\right) v$, which implies that $V\left(\phi_{t}\left(x_{1}, g\right)-\phi_{t}\left(x_{2}, g\right), g \cdot t\right)<V\left(x_{1}-x_{2}, g\right)$ for all $t>0$. Thus, the equality in (3.6) holds if and only if $x_{1}-x_{2} \in \operatorname{span}\{v\}$, which completes our proof.

Remark 3.9. By virtue of (3.9), we note that the orbit difference $\left\{\phi_{t}\left(x_{1}, g\right)-\right.$ $\left.\phi_{t}\left(x_{2}, g\right): t \geq 0\right\}$ is bounded, although the orbits $\mathcal{O}^{+}\left(x_{i}, g\right)$ themselves, $i=1,2$, may not necessarily be bounded.

Proof of Theorem A. Before giving the proof of property (3.3), we show how this asymptotic property helps us to deduce the uniqueness of the 1-cover w.r.t. $\tilde{\Pi}$, as well as the 1-cover property of $\tilde{\omega}(\tilde{x}, g)$ for every bounded orbit $\tilde{O}^{+}(\tilde{x}, g)$.

Indeed, let $K_{i}=\left\{\left(\tilde{x}_{g}^{i}, g\right): g \in Y\right\}, i=1,2$, be two 1-covers of $Y$ with respect to $\tilde{\Pi}$. Suppose that there exists a $g^{*} \in Y$ such that $\tilde{x}_{g^{*}}^{1} \neq \tilde{x}_{g^{*}}^{2}$. Then, choose a sequence $t_{n}$ such that $g^{*} \cdot t_{n} \rightarrow g^{*}$ as $n \rightarrow \infty$. By property (3.3), one has

$$
0 \neq\left\|\tilde{x}_{g^{*}}^{1}-\tilde{x}_{g^{*}}^{2}\right\|=\lim _{n \rightarrow \infty}\left\|\tilde{x}_{g^{*} \cdot t_{n}}^{1}-\tilde{x}_{g^{*} \cdot t_{n}}^{2}\right\|=\lim _{n \rightarrow \infty}\left\|\tilde{\phi}_{t_{n}}\left(\tilde{x}_{g^{*}}^{1}, g^{*}\right)-\tilde{\phi}_{t_{n}}\left(\tilde{x}_{g^{*}}^{1}, g^{*}\right)\right\|=0,
$$

a contradiction. So $K_{1}=K_{2}$, which leads to the uniqueness of the 1-cover.

Now, for any $(\tilde{x}, g) \in X_{0} \times Y$ with bounded orbit $\tilde{\mathcal{O}}^{+}(\tilde{x}, g)$, suppose that there exists a $g_{0} \in Y$ such that on this fiber one can find two distinct points from its 
omega-limit set, i.e., $\left(\tilde{z}_{1}, g_{0}\right),\left(\tilde{z}_{2}, g_{0}\right) \in \tilde{\omega}(\tilde{x}, g) \cap p^{-1}\left(g_{0}\right)$. It then follows from (3.3) that

$$
\left\|\tilde{\phi}_{t}\left(\tilde{z}_{1}, g_{0}\right)-\tilde{\phi}_{t}\left(\tilde{z}_{2}, g_{0}\right)\right\| \rightarrow 0 \quad \text { as } t \rightarrow \infty
$$

which implies that $\left(\tilde{z}_{1}, g_{0}\right)$ and $\left(\tilde{z}_{2}, g_{0}\right)$ are not a positively fiber distal pair of the skew-product semiflow $\tilde{\Pi}$. This contradicts Lemma 3.7. Therefore, $\tilde{\omega}(\tilde{x}, g)$ is a 1-cover of $Y$ with respect to $\tilde{\Pi}$. So it remains to prove the property (3.3).

Suppose on the contrary that there exist two points $\left(\tilde{x}_{i}, g\right), i=1,2$, with their orbits $\tilde{\mathcal{O}}^{+}\left(\tilde{x}_{i}, g\right)$ bounded, such that (3.3) does not hold. Then, by Lemma 3.6. one can choose a sequence $t_{n} \rightarrow \infty$ such that $g \cdot t_{n} \rightarrow g^{*} \in Y$ and $\tilde{\phi}_{t_{n}}\left(\tilde{x}_{i}, g\right) \rightarrow x_{i}^{*}, i=$ 1,2 , as $n \rightarrow \infty$. Here $x_{1}^{*}, x_{2}^{*} \in X_{0}$ and $x_{1}^{*} \neq x_{2}^{*}$.

Now pick some $x_{i} \in \pi^{-1}\left(\tilde{x}_{i}\right), i=1,2$. It follows from Proposition 3.1 that

$$
\pi \phi_{t_{n}}\left(x_{i}, g\right)=\tilde{\phi}_{t_{n}}\left(\tilde{x}_{i}, g\right) \rightarrow x_{i}^{*}, \quad i=1,2,
$$

as $n \rightarrow \infty$. By the definition of $\pi$,

$$
\begin{aligned}
& \phi_{t_{n}}\left(x_{1}, g\right)-\phi_{t_{n}}\left(x_{2}, g\right) \\
& =\pi \phi_{t_{n}}\left(x_{1}, g\right)-\pi \phi_{t_{n}}\left(x_{2}, g\right)+\left\langle f, \phi_{t_{n}}\left(x_{1}, g\right)-\phi_{t_{n}}\left(x_{2}, g\right)\right\rangle v
\end{aligned}
$$

Fix a $\tau>0$; it follows from (A3) that

$$
\begin{aligned}
& \phi_{\tau}\left(\phi_{t_{n}}\left(x_{1}, g\right)-\phi_{t_{n}}\left(x_{2}, g\right), g \cdot t_{n}\right) \\
& =\phi_{\tau}\left(\pi \phi_{t_{n}}\left(x_{1}, g\right)-\pi \phi_{t_{n}}\left(x_{2}, g\right), g \cdot t_{n}\right)+\left\langle f, \phi_{t_{n}}\left(x_{1}, g\right)-\phi_{t_{n}}\left(x_{2}, g\right)\right\rangle v .
\end{aligned}
$$

By eliminating $v$ from (3.11) and (3.12), one has

$$
\begin{aligned}
& \phi_{t_{n}}\left(x_{1}, g\right)-\phi_{t_{n}}\left(x_{2}, g\right)=\pi \phi_{t_{n}}\left(x_{1}, g\right)-\pi \phi_{t_{n}}\left(x_{2}, g\right) \\
& +\phi_{\tau}\left(\phi_{t_{n}}\left(x_{1}, g\right)-\phi_{t_{n}}\left(x_{2}, g\right), g \cdot t_{n}\right)-\phi_{\tau}\left(\pi \phi_{t_{n}}\left(x_{1}, g\right)-\pi \phi_{t_{n}}\left(x_{2}, g\right), g \cdot t_{n}\right) .
\end{aligned}
$$

Note that $\Pi$ is completely continuous and $\left\{\phi_{t_{n}}\left(x_{1}, g\right)-\phi_{t_{n}}\left(x_{2}, g\right)\right\}$ is bounded by Remark 3.9. Then there exists a subsequence of $\left\{t_{n}\right\}$, still denoted by $\left\{t_{n}\right\}$, such that $\phi_{\tau}\left(\phi_{t_{n}}\left(x_{1}, g\right)-\phi_{t_{n}}\left(x_{2}, g\right), g \cdot t_{n}\right)$ converges as $n \rightarrow \infty$. Together with (3.10) and (3.13), it yields that

$$
\phi_{t_{n}}\left(x_{1}, g\right)-\phi_{t_{n}}\left(x_{2}, g\right) \rightarrow \eta \quad \text { as } n \rightarrow \infty .
$$

Back to (3.11), we obtain that

$$
\phi_{t_{n}}\left(x_{1}, g\right)-\phi_{t_{n}}\left(x_{2}, g\right) \rightarrow x_{1}^{*}-x_{2}^{*}+\langle f, \eta\rangle v \quad \text { as } n \rightarrow \infty
$$

On the other hand, given any $t>0$, it holds that

$$
\begin{aligned}
& \phi_{t_{n}+t}\left(x_{1}, g\right)-\phi_{t_{n}+t}\left(x_{2}, g\right) \\
= & \phi_{t}\left(\phi_{t_{n}}\left(x_{1}, g\right), g \cdot t_{n}\right)-\phi_{t}\left(\phi_{t_{n}}\left(x_{2}, g\right), g \cdot t_{n}\right) \\
= & \phi_{t}\left(\pi \phi_{t_{n}}\left(x_{1}, g\right), g \cdot t_{n}\right)-\phi_{t}\left(\pi \phi_{t_{n}}\left(x_{2}, g\right), g \cdot t_{n}\right) \\
& +\left\langle f, \phi_{t_{n}}\left(x_{1}, g\right)-\phi_{t_{n}}\left(x_{2}, g\right)\right\rangle v .
\end{aligned}
$$

It then follows from (3.14) and (3.10) that

$$
\phi_{t_{n}+t}\left(x_{1}, g\right)-\phi_{t_{n}+t}\left(x_{2}, g\right) \rightarrow \phi_{t}\left(x_{1}^{*}, g^{*}\right)-\phi_{t}\left(x_{2}^{*}, g^{*}\right)+\langle f, \eta\rangle v
$$

as $n \rightarrow \infty$.

Now, by virtue of Lemma 3.8, we note that the continuous function

$$
s \mapsto V\left(\phi_{s}\left(x_{1}, g\right)-\phi_{s}\left(x_{2}, g\right), g \cdot s\right)
$$


converges decreasingly as $s \rightarrow \infty$. By letting $t_{n} \rightarrow \infty$ in (3.15) and (3.16) it then follows that

$$
V\left(\phi_{t}\left(x_{1}^{*}, g^{*}\right)-\phi_{t}\left(x_{2}^{*}-\langle f, \theta\rangle v, g^{*}\right), g^{*} \cdot t\right)=V\left(x_{1}^{*}-\left(x_{2}^{*}-\langle f, \theta\rangle v\right), g^{*}\right)
$$

for any $t>0$. According to Lemma 3.8(ii), this indicates

$$
x_{1}^{*}-x_{2}^{*}+\langle f, \theta\rangle v \in \operatorname{span}\{v\}
$$

and hence $x_{1}^{*}-x_{2}^{*} \in \operatorname{span}\{v\}$. Note that $x_{1}^{*}, x_{2}^{*} \in X_{0}$ and $x_{1}^{*} \neq x_{2}^{*}$, a contradiction. Thus we have proved (3.3), which completes the proof of Theorem A.

Proof of Theorem B. 1) Assume that $\mathcal{O}^{+}(x, g)$ is bounded. Then it is relatively compact because $\Pi$ is completely continuous. As a consequence, $\omega(x, g)$ is a minimal set which admits a fiber distal flow extension by Lemma 2.3 and Lemma 3.3 . Suppose that $\omega(x, g)$ is not a 1 -cover of $Y$. Then there exist two distinct points $\left(x_{1}, g_{0}\right),\left(x_{2}, g_{0}\right) \in \omega(x, g)$. By virtue of the property (3.3), one can find a sequence $t_{n} \rightarrow \infty$ such that

$$
\lim _{n \rightarrow \infty}\left[\phi_{t_{n}}\left(x_{1}, g_{0}\right)-\phi_{t_{n}}\left(x_{2}, g_{0}\right)\right] \in \operatorname{span}\{v\} \quad \text { as } n \rightarrow \infty .
$$

Note that $\omega(x, g)$ is totally invariant and compact. One can assume without loss of generality that $\phi_{t_{n}}\left(x_{i}, g_{0}\right) \rightarrow\left(x_{i}^{*}, g^{*}\right) \in \omega(x, g)$ as $n \rightarrow \infty$, for $i=1,2$. It then follows from (3.17) and the fiber-distal property of $\omega(x, g)$ that $\left(x_{1}^{*}, g^{*}\right)$ and $\left(x_{2}^{*}, g^{*}\right)$ are two distinct points related by "«" on the same fiber. This contradicts Lemma 2.4. Hence $\omega(x, g)$ is a 1-cover of $Y$ w.r.t. $\Pi$.

2 ) It is a direct corollary by Remark 3.2 and Theorem A.

\section{Time-Dependent ChEmical REACtion NETWORKS}

Due to the challenges posed by molecular and systems biology, the investigation of the asymptotical behavior of chemical reaction networks is an area of growing interest. In this section, we will utilize our main theoretical results to establish the global dynamics of time-dependent chemical reaction networks.

A chemical reaction network is a list of chemical reactions $\mathcal{R}_{i}, i=1, \cdots, n$, which specify how certain combinations of chemical species are converted into other combinations of chemical species. Let $S_{j}$ be the $j$-th chemical species for $j=$ $1, \cdots, m$. The $i$-th chemical reaction $\mathcal{R}_{i}$ can be written as

$$
\mathcal{R}_{i}: \sum_{j=1}^{m} \alpha_{i j} S_{j} \rightarrow \sum_{j=1}^{m} \beta_{i j} S_{j} \quad \text { (called irreversible reactions) }
$$

or

$$
\mathcal{R}_{i}: \sum_{j=1}^{m} \alpha_{i j} S_{j} \leftrightarrow \sum_{j=1}^{m} \beta_{i j} S_{j} \quad \text { (called reversible reactions), }
$$

where $\alpha_{i j}, \beta_{i j}$ are nonnegative integers called stoichiometry coefficients. For convenience we arrange these coefficients in a matrix, called a stoichiometry matrix $M$, defined as

$$
M_{i, j}=\alpha_{i j}-\beta_{i j}, \quad \text { for } 1 \leq i \leq n \text { and } 1 \leq j \leq m .
$$

Let $U_{j}$ be the concentration of the $j$-th reaction species $S_{j}$, and define $U=$ $\left(U_{1}, U_{2}, \cdots, U_{m}\right)$ as the $m$-vector of the species concentrations. Since each chemical reaction $\mathcal{R}_{i}$ takes place continuously in time with its own reaction rate $f_{i}$ affected by the concentrations of different species, we write the $n$-vector of reaction rates $f(t, U)=\left(f_{1}(t, U), f_{2}(t, U), \cdots, f_{n}(t, U)\right)$. 
The most popular functional form of $f_{i}(t, U)$ found in the literature are mass action kinetics, Michaelis-Menten (or Monod) kinetics and Hill kinetics, which in case of an irreversible reaction are given by the following specific form:

$$
\kappa_{i} \prod_{j: \alpha_{i j}>0} U_{j}^{\alpha_{i j}}, \quad m_{i} \prod_{j: \alpha_{i j}>0} \frac{U_{j}}{U_{j}+a_{j}}, \quad D_{i} \prod_{j: \alpha_{i j}>0} \frac{\left(U_{j}\right)^{j}}{\left(U_{j}\right)^{j}+b_{j}},
$$

respectively. Here the reaction parameters $k_{i}, m_{i}, a_{j}, D_{i}, b_{j}$ are positive and depend on time $t$.

In practical laboratory experiments the system usually evolves, influenced by external time-dependent effects which are periodic or roughly periodic. For instance, in the case of mass action kinetics, the reaction coefficients $\kappa_{i}(t)$ are time-periodic or may exhibit different, noncommensurate periods. As a consequence, one may assume that $\kappa_{i}(t)$ are time-recurrent functions in time $t$. With the above notation, a chemical reaction network is described by the following differential equations:

$$
\left\{\begin{array}{l}
\dot{U}=M f(t, U), \\
U(0)=U_{0} \in \mathbb{R}_{+}^{m},
\end{array}\right.
$$

where $\mathbb{R}_{+}^{m}=\left\{U \in \mathbb{R}^{m}: U_{i} \geq 0\right\}$. Of course, $U_{0}$ is the initial concentration of all species and $f(t, U)$ is a time-recurrent vector-valued function. In general, by using certain conversation laws (see, e.g., 3, 26]), one may assume that every solution of (4.1) is bounded.

Given a chemical reaction network (4.1), we introduce the so-called associate system in reaction coordinates (see, e.g., [4, 37]). For any $\sigma \in \mathbb{R}_{+}^{m}$, such a system in reaction coordinates is defined as the following nonautonomous system:

$$
\left\{\begin{array}{l}
\dot{u}=F_{\sigma}(t, u), \quad t>0 \\
u(0)=u_{0} \in \mathbb{R}^{n}
\end{array}\right.
$$

where $F_{\sigma}(t, u)=f(t, \sigma+M u)$ is time-recurrent. Here $u=\left(u_{1}, \cdots, u_{n}\right)$ is called the extent of the reaction (see [37]).

For systems (4.2) and (4.1), let $H\left(F_{\sigma}\right)$ and $H(f)$ be the hull of $F_{\sigma}$ and $f$, respectively. Then $H\left(F_{\sigma}\right)$ and $H(f)$ are minimal because $f$ is time-recurrent. Moreover, there is a flow isomorphism from $H\left(F_{\sigma}\right)$ to $H(f)$. As a consequence, for any $g \in H\left(F_{\sigma}\right)$ there exists a unique $h \in H(f)$ such that

$$
g(t, u)=h(t, \sigma+M u) .
$$

In particular, $g=F_{\sigma}$ if and only if $h=f$.

For every $g \in H\left(F_{\sigma}\right)$ and $h \in H(f)$ in (4.3), let $U\left(t ; U_{0}, h\right)$ and $u\left(t ; u_{0}, g\right)$ be the solutions of

$\left.(4.1\}_{h}\right)$

$$
\left\{\begin{array}{l}
\dot{U}=M h(t, U), \quad t>0 \\
U(0)=U_{0} \in \mathbb{R}_{+}^{m}
\end{array}\right.
$$

and

$$
\left\{\begin{array}{l}
\dot{u}=g(t, u), \quad t>0 \\
u(0)=u_{0} \in \mathbb{R}^{n},
\end{array}\right.
$$

respectively.

The following lemma shows an important relation between the solutions $U\left(t ; U_{0}, h\right)$ and $u\left(t ; u_{0}, g\right)$. As a consequence, at least in principle, the dynamics of (4.1b) can be understood by studying the dynamics of $4.2 \mathrm{~b}$ ). 
Lemma 4.1. Let $U\left(t ; U_{0}, h\right)$ and $u\left(t ; u_{0}, g\right)$ be the solutions of (4.1b) and (4.2. respectively. If the initial value $U_{0}=\sigma+M u_{0}$, then

$$
U\left(t ; U_{0}, h\right)=\sigma+M u\left(t ; u_{0}, g\right) \quad \text { for } t \geq 0 .
$$

Proof. We use similar arguments as in [4. Since $U\left(t ; U_{0}, h\right)$ is bounded (hence defined on all $t \geq 0$ ), we define

$$
\widehat{u}(t):=u_{0}+\int_{0}^{t} h\left(\tau, U\left(\tau ; U_{0}, h\right)\right) \mathrm{d} \tau
$$

and

$$
N(t):=\sigma+M \widehat{u}(t)=\left(\sigma+M u_{0}\right)+\int_{0}^{t} M h\left(\tau, U\left(\tau, U_{0}, h\right)\right) \mathrm{d} \tau
$$

for all $t \geq 0$. By differentiating $N$, it yields that $d N / d t=M h\left(t, U\left(t, U_{0}, h\right)\right)$. So $d N / d t=d U\left(t ; U_{0}, h\right) / d t$, and hence $N(t)=U\left(t, U_{0}, h\right)+C, t \geq 0$, for some constant $C$. By virtue of (4.4) and our initial value assumption, we have $N(0)=\sigma+M u_{0}=$ $U_{0}=U\left(0 ; U_{0}, h\right)$. It then follows that

$$
U\left(t ; U_{0}, h\right)=N(t)=\sigma+M \widehat{u}(t)
$$

for all $t \geq 0$, and consequently

$$
\widehat{u}(t)=\widehat{u}(0)+\int_{0}^{t} h(\tau, \sigma+M \widehat{u}(\tau)) \mathrm{d} \tau .
$$

Noticing (4.3), we have $\widehat{u}(t)=\widehat{u}(0)+\int_{0}^{t} g(\tau, \widehat{u}(\tau)) \mathrm{d} \tau$, which implies that $\widehat{u}(t)$ is a solution of (4.2 $u\left(t ; u_{0}, g\right)$, and hence, by (4.5),

$$
U\left(t ; U_{0}, h\right)=\sigma+M u\left(t ; u_{0}, g\right)
$$

for all $t \geq 0$. Thus we have completed the proof.

For the new introduced system (4.2,

$$
X_{\sigma}^{n}=\left\{u \in \mathbb{R}^{n}: \sigma+M u \geq 0\right\} .
$$

It then follows from Lemma 4.1 that one can define a skew-product flow associated with (4.26) by $\Pi: X_{\sigma}^{n} \times H\left(F_{\sigma}\right) \times \mathbb{R}^{+} \rightarrow X_{\sigma}^{n} \times H\left(F_{\sigma}\right)$,

$$
\Pi\left(t, u_{0}, g\right)=\left(u\left(t ; u_{0}, g\right), g \cdot t\right) .
$$

Remark 4.2. The main reason for lifting the nonmonotone chemical reaction network (4.1) to the new system (4.2] is that quite surprisingly, in many examples, including very large ones taken from the current biochemical literature (e.g., the benchmark models in the area of the phosphorylation/dephosphorylation process, and more complex reaction networks which arise in many signal transduction pathways, the futile cycle and the MAPK cascade (see [12, 26, 41, 4, 9])), the skewproduct flow $\Pi$ of the new system description in reaction coordinates turns out to be strongly monotone. As an illustrated example, we will show at the end of this section the technical detail of verifying the monotonicity for a simple phosphorylation process. A more general graph-theoretic approach for the monotonicity can be found in the recent work [3]. 
According to Remark 4.2, in the following we will thoroughly analyze the skewproduct flow $\Pi$ associated with the new system (4.2 $\mathrm{g}$ ) under the assumption that $\Pi$ is strongly monotone.

Now, by choosing any $v \in \operatorname{Ker} M$ with $\|v\|=1$, we can define the phasetranslation group $G$ with respect to $v$,

$$
a: X_{\sigma}^{n} \rightarrow X_{\sigma}^{n} ; a \cdot u:=u+a v,
$$

by any scalar $a \in \mathbb{R}$ acting on $X_{\sigma}^{n}$.

The following proposition indicates that $\Pi$ satisfies (A3) in Section 3:

Proposition 4.3. The group $G$ commutes with $\Pi$, i.e., $u\left(t ; g, a \cdot u_{0}\right)=a \cdot u\left(t ; g, u_{0}\right)$, for any $\left(u_{0}, g\right) \in X_{\sigma}^{n} \times H\left(F_{\sigma}\right), t \geq 0$, and $a \in G$.

Proof. By uniqueness of the solutions, it suffices to show that $a \cdot u\left(t ; g, u_{0}\right)$ is a solution of (4.21). To end this, choose any $g \in H\left(F_{\sigma}\right)$. It follows from (4.3) that there exists some $h \in H(f)$ such that $g(t, u)=h(t, \sigma+M u)$. Consequently,

$$
\begin{aligned}
\frac{\mathrm{d}\left(a \cdot u\left(t ; g, u_{0}\right)\right)}{\mathrm{d} t} & =\frac{\mathrm{d}\left(u\left(t ; g, u_{0}\right)+a v\right)}{\mathrm{d} t}=\frac{\mathrm{d} u\left(t ; g, u_{0}\right)}{\mathrm{d} t}=g\left(t, u\left(t ; g, u_{0}\right)\right) \\
& =h\left(t, \sigma+M u\left(t ; g, u_{0}\right)\right) \\
& =h\left(t, \sigma+M\left(u\left(t ; g, u_{0}\right)+a v\right)\right) \\
& =g\left(t, u\left(t ; g, u_{0}\right)+a v\right)=g\left(t, a \cdot u\left(t ; g, u_{0}\right)\right),
\end{aligned}
$$

for each $a \in G$. We have completed the proof.

Remark 4.4. Although the skew-product flow $\Pi$ has been known to be strongly monotone and $G$-invariant with respect to the phase-translation group, we ignore that a priori there is no guarantee that solutions of (4.2b) are bounded, and hence no guarantee for the boundedness of the orbit of $\Pi$ as well. (Notice that this is different from the case in the chemical reaction network (4.1), where the boundedness of the solutions is fully guaranteed by certain conservation laws.) As pointed out by Angeli et al. in [3, p. 596]: "this issue constitutes the main technical difficulty that needs to be surmounted in order for us to obtain the convergence results for system (4.1)". In order to resolve such a problem, we introduced the so-called pseudo-boundedness of the orbits of $\Pi$ in the previous section.

The following proposition shows that the orbits of $\Pi$ are actually pseudo-bounded if the kernel of the stoichiometry matrix $M$ intersects the interior of $\mathbb{R}_{+}^{n}$ :

Proposition 4.5. Assume that $v \in \operatorname{Ker} M \cap \operatorname{Int} \mathbb{R}_{+}^{n} \neq \emptyset$ with $\|v\|=1$. Let $\pi$ be defined as in (2.4). Then, for any $\left(u_{0}, g\right) \in X_{\sigma}^{n} \times H\left(F_{\sigma}\right)$, the orbit $\mathcal{O}^{+}\left(u_{0}, g\right)$ of $\Pi$ in (4.6) is pseudo-bounded.

Proof. Given any $\left(u_{0}, g\right) \in X_{\sigma}^{n} \times H\left(F_{\sigma}\right)$, it then follows from Lemma 4.1 that there exist some $h \in H(f)$ such that

$$
U\left(t ; U_{0}, h\right)=\sigma+M u\left(t ; u_{0}, g\right) \quad \text { for all } t \geq 0 .
$$

Since $U\left(t ; U_{0}, h\right)$ is bounded for all $t \geq 0, M u\left(t ; u_{0}, g\right)$ is bounded for all $t \geq 0$.

It is easy to see that we are done if we proved the following claim: $\mathcal{O}^{+}\left(u_{0}, g\right)$ is pseudo-bounded if and only if $M u\left(t ; u_{0}, g\right)$ is bounded for all $t \geq 0$. Indeed, on the one hand, note that

$$
M \pi u=M(u-\langle f, u\rangle v)=M u-\langle f, u\rangle M v=M u
$$


for any $u \in \mathbb{R}^{n}$. So, the pseudo-boundedness of $\mathcal{O}^{+}\left(u_{0}, g\right)$ implies the boundedness of $M u\left(t ; u_{0}, g\right)$. On the other hand, let $Q=\left.M\right|_{X_{0}}$ be the restriction of $M$ to the space $X_{0}$. Since $\operatorname{Ker} M=\operatorname{span}\{v\}$, it is easy to see that $Q: X_{0} \rightarrow \operatorname{Range} M \subset \mathbb{R}^{m}$ is a one-to-one map. Moreover, both $Q$ and $\left.Q^{-1}\right|_{\operatorname{Range} M}$ are Lipschitz. As a consequence, $Q^{-1} M u=Q^{-1} M \pi u=Q^{-1} Q \pi u=\pi u$, for all $u \in \mathbb{R}^{n}$. Moreover, the Lip-property of $\left.Q^{-1}\right|_{\text {Range } M}$ implies that $\pi u\left(t ; u_{0}, g\right)$ is bounded if $M u\left(t ; u_{0}, g\right)$ is bounded. Thus we have proved the claim, which completes our proof.

Based on our main results in Section 3, one can show the global convergence for the chemical reaction network (4.1) with time-recurrent kinetics. For simplicity, here we only present the convergence result with time almost-periodic (almostautomorphic) kinetics.

Theorem 4.6. Let $U(t)$ be any solution, with the initial value $U_{0} \in \mathbb{R}_{+}^{m}$, of the time almost-periodic (almost-automorphic) chemical reaction network (4.1) with $\operatorname{Ker} M \cap \operatorname{Int} \mathbb{R}_{+}^{n} \neq \emptyset$. Assume that the skew-product flow $\Pi$ associated with system (4.21) in reaction coordinates is strongly monotone. Then $U(t)$ is asymptotic to an almost-periodic (almost-automorphic) solution $U^{*}(t)$ of (4.1) with its frequency module $\mathcal{M}\left(U^{*}\right) \subseteq \mathcal{M}(f)$.

In particular, if $f$ in (4.1) is periodic (quasi-periodic) in time $t$, then $U(t)$ is asymptotic to a periodic (quasi-periodic) solution $U^{*}(t)$ of (4.1).

Proof. By virtue of Proposition 4.3 and our assumption, the skew-product flow $\Pi$ (see (4.6) ), associated with system (4.2b), satisfies the assumptions (A1)-(A3) in Section 3.

Note also that $\operatorname{Ker} M \cap \operatorname{Int} \mathbb{R}_{+}^{n} \neq \emptyset$. It then follows from Proposition 4.5 that the orbit $\mathcal{O}^{+}\left(u_{0}, g\right)$ of $\Pi$ is pseudo-bounded for any $\left(u_{0}, g\right) \in X_{\sigma}^{n} \times H\left(F_{\sigma}\right)$. In particular, choose $\left(u_{0}=0, g=F_{\sigma}\right) \in X_{\sigma}^{n} \times H\left(F_{\sigma}\right)$. Then Theorem B implies that the omega-limit set of induced orbit $\tilde{\mathcal{O}}^{+}\left(0, F_{\sigma}\right)$ is a 1-cover of $H\left(F_{\sigma}\right)$. That is to say, $\pi u\left(t ; 0, F_{\sigma}\right)$ is asymptotic to an almost-periodic (almost-automorphic) function $w_{\sigma}^{*}(t)$ with its module $\mathcal{M}\left(w_{\sigma}^{*}\right) \subseteq \mathcal{M}\left(F_{\sigma}\right)$. Now choose $\sigma \in \mathbb{R}_{+}^{m}$ such that $\sigma=U_{0}=$ $\sigma+M \cdot 0$. It follows from (4.7) and Lemma 4.1 that $U(t)=\sigma+M u\left(t ; 0, F_{\sigma}\right)=$ $\sigma+M \pi u\left(t ; 0, F_{\sigma}\right)=U_{0}+M \pi u\left(t ; 0, F_{\sigma}\right)$. Therefore, $U(t)$ will be asymptotic to an almost periodic (almost automorphic) solution $U^{*}(t)=U_{0}+M w_{\sigma}^{*}(t)$ of (4.1) with its module $\mathcal{M}\left(U^{*}\right) \subseteq \mathcal{M}\left(w_{\sigma}^{*}\right) \subseteq \mathcal{M}\left(F_{\sigma}\right) \subseteq \mathcal{M}(f)$.

In particular, if $f$ in (4.1) is periodic (quasi-periodic) in time $t$, then $U(t)$ is asymptotic to a periodic (quasi-periodic) solution $U^{*}(t)$ of (4.1). We have completed the proof.

As mentioned in Remark 4.2, there are large quantities of models taken from the current biochemical literature admitting the monotonicity of the new system description in reaction coordinates. The benchmark examples include the phosphorylation/dephosphorylation processes (sometimes called enzyme futile cycles; see, e.g., 12, 26, 41, 4), nonmass action kinetics under the QSSA assumption in the dimerization reactions of proteins (see [26]) and more complex reaction networks which arise in many signal transduction pathways, the MAPK cascade and the RKIP inhibited ERK pathway from Cho et al. 9].

Accordingly Theorem 4.6 implies the global dynamics of all the above-mentioned time-dependent benchmark chemical reaction networks. In the following, we do not 
intend to present them all in detail, but choose a simple phosphorylation/dephosphorylation process in mass action kinetics as an illustrated example to show the technical detail of verifying the monotonicity for the new system in reaction coordinates.

- Simple phosphorylation/dephosphorylation (Enzymatic futile cycle). The model is diagrammed in Figure 1. Such a cycle occurs when two metabolic path-

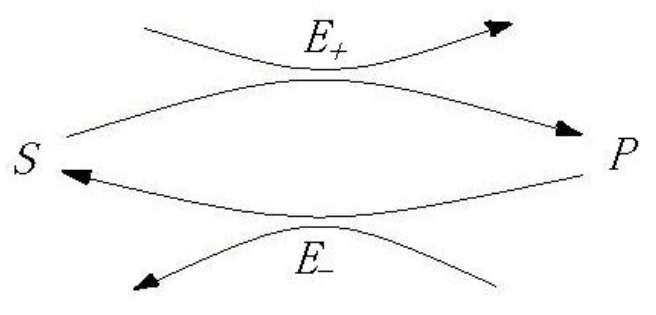

Figure 1. Enzymatic futile cycle reaction mechanism.

ways run simultaneously in opposite directions and have no overall effect other than to dissipate energy in the form of heat (see, e.g., [41, 48]). In Figure 1, $E_{+}, E_{-}$denote the forward and reverse (e.g., activating and deactivating) enzymes, and $S, P$ stand for the concentrations of the forward substrate and product, respectively. Enzymatic futile cycles and cycle cascades represent a recurring control theme in biological molecular networks, appearing in a wide variety of processes from energy metabolism to signal transduction (see 11, 27, 7, 15, 50, 28, 49]).

The representation for such a model, illustrated in Figure 1, is

$$
\begin{aligned}
& S+E_{+} \leftrightarrow C_{+} \rightarrow P+E_{+}, \\
& P+E_{-} \leftrightarrow C_{-} \rightarrow S+E_{-},
\end{aligned}
$$

where the intermediate complex, $C_{+}$or $C_{-}$, dissociates either back into the original reactants or into the product and the enzyme. Denote concentrations with the same letters as the substrates or enzymes. The well-mixed mass-action kinetics model of such futile cycle is obtained as (see [4, 3])

$$
\left\{\begin{array}{l}
\dot{S}=-\kappa_{1}(t) E_{+} S+\kappa_{-1}(t) C_{+}+\kappa_{4}(t) C_{-}, \\
\dot{P}=\kappa_{2}(t) C_{+}-\kappa_{3}(t) E_{-} P+\kappa_{-3}(t) C_{-}, \\
\dot{E}_{+}=-\kappa_{1}(t) E_{+} S+\kappa_{-1}(t) C_{+}+\kappa_{2}(t) C_{+}, \\
\dot{E}_{-}=-\kappa_{3}(t) E_{-} P+\kappa_{-3}(t) C_{-}+\kappa_{4}(t) C_{-}, \\
\dot{C}_{+}=\kappa_{1}(t) E_{+} S-\kappa_{-1}(t) C_{+}-\kappa_{2}(t) C_{+} \\
\dot{C}_{-}=\kappa_{3}(t) E_{-} P-\kappa_{-3}(t) C_{-}-\kappa_{4}(t) C_{-}
\end{array}\right.
$$

Here $\kappa_{i}(t), i= \pm 1,2, \pm 3,4$, are time-dependent reaction coefficients which quantify the speed of the different reactions. For more generality, we assume that $\kappa_{i}(t)$ are almost-periodic (almost-automorphic) functions in time $t$. We also assume that all the $\kappa_{i}(t)$ are uniformly positive, i.e., there exists a $\delta>0$ such that $\kappa_{i}(t) \geq \delta$ for all $i= \pm 1,2, \pm 3,4$ and $t \geq 0$. 
Now we rewrite equations (4.8) in a standard form of system (4.1) with $m=6$ for representing the chemical reaction network, in which

$$
U=\left(P, Q, E_{+}, E_{-}, C_{+}, C_{-}\right)^{T} \in \mathbb{R}_{+}^{6}
$$

is the species vector, and $f(t, U)$ and $M$ are the reaction rates vector and the stoichiometry matrix, respectively:

$$
f(t, U)=\left(\begin{array}{c}
\kappa_{1}(t) E_{+} S-\kappa_{-1}(t) C_{+} \\
\kappa_{2}(t) C_{+} \\
\kappa_{3}(t) E_{-} P-\kappa_{-3}(t) C_{-} \\
\kappa_{4}(t) C_{-}
\end{array}\right), \quad M=\left(\begin{array}{cccc}
-1 & 0 & 0 & 1 \\
0 & 1 & -1 & 0 \\
-1 & 1 & 0 & 0 \\
0 & 0 & -1 & 1 \\
1 & -1 & 0 & 0 \\
0 & 0 & 1 & -1
\end{array}\right) .
$$

Along each solution, one can easily see that $S(t)+P(t)+C_{+}(t)+C_{-}(t), E_{+}(t)+$ $C_{+}(t)$ and $E_{-}(t)+C_{-}(t)$ satisfy the conservation law: such three quantities are invariant with respect to time $t$. Together with the fact that all the components of $U$ are nonnegative, the conservation law implies that every solution $U(t)$ of (4.1) is bounded.

Now we change the system (4.1) to the new associated system (4.2) with $n=4$ in reaction variables, for which

$$
F_{\sigma}(t, u)=\left(\begin{array}{c}
\kappa_{1}(t)\left(\sigma_{3}+u_{2}-u_{1}\right)\left(\sigma_{1}+u_{4}-u_{1}\right)-\kappa_{-1}(t)\left(\sigma_{5}+u_{1}-u_{2}\right) \\
\kappa_{2}(t)\left(\sigma_{5}+u_{1}-u_{2}\right) \\
\kappa_{3}(t)\left(\sigma_{4}+u_{4}-u_{3}\right)\left(\sigma_{2}+u_{2}-u_{3}\right)-\kappa_{-3}(t)\left(\sigma_{6}+u_{3}-u_{4}\right) \\
\kappa_{4}(t)\left(\sigma_{6}+u_{3}-u_{4}\right)
\end{array}\right)
$$

with $\sigma=\left(\sigma_{1}, \cdots, \sigma_{6}\right) \in \mathbb{R}_{+}^{6}$.

Note that $v=\left(\frac{1}{4}, \frac{1}{4}, \frac{1}{4}, \frac{1}{4}\right) \in \operatorname{Ker} M \cap \operatorname{Int} \mathbb{R}_{+}^{4} \neq \emptyset$. Then, in order to utilize Theorem 4.6 to obtain the convergence result for the futile cycle (4.8), one only needs to show the strong monotonicity of the skew-product flow $\Pi$ associated with the system (4.26).

To this end, for each $g \in H\left(F_{\sigma}\right)$, a direct calculation yields that the Jacobian $\frac{\partial g}{\partial u}$ of $g$ has the form

$$
\left(\begin{array}{cccc}
- & + & 0 & \kappa_{1}^{g}(t)\left(\sigma_{3}+\left(u_{2}-u_{1}\right)\right) \\
+ & - & 0 & 0 \\
0 & \kappa_{3}^{g}(t)\left(\sigma_{4}+\left(u_{4}-u_{3}\right)\right) & - & + \\
0 & 0 & + & -
\end{array}\right),
$$

where $\kappa_{i}^{g}(t), i=1,3$, are the corresponding parts of $\kappa_{i}(t)$ with respect to $g \in$ $H\left(F_{\sigma}\right)$. It is easy to see that $\kappa_{i}^{g}(t)$ is uniformly-positive as well. Moreover, in (4.9), "-" represents strictly negative elements and "+" means strictly positive elements. Therefore, $\Pi$ is monotone in the sense of Definition 2.1. Furthermore, we have

Lemma 4.7. $\Pi$ is strongly monotone if the Jacobian matrix $\frac{\partial g}{\partial u}(t, u)$ is irreducible almost everywhere along each solution of (4.2, $)$.

Proof. Let $u(t)$ and $v(t)$ be solutions of (4.2b) with initial data $u_{0}$ and $v_{0}\left(u_{0}>v_{0}\right)$, respectively. Let $w(t):=u(t)-v(t)$. Then

$$
\left\{\begin{array}{l}
\dot{w}(t)=A(t) w(t), \quad t \geq 0 \\
w(0)=w_{0}>0
\end{array}\right.
$$


where $A(t)=\int_{0}^{1} \frac{\partial g}{\partial u}(t, \lambda u(t)+(1-\lambda) v(t)) \mathrm{d} \lambda$. Note that every element of $\frac{\partial g}{\partial u}(t, u)$ is linear with respect to $u$. It then follows that $A(t)=\frac{1}{2}\left(\frac{\partial g}{\partial u}(t, u(t))+\frac{\partial g}{\partial u}(t, v(t))\right)$. So, $A(t)$ is irreducible almost everywhere in $t>0$ because $\frac{\partial g}{\partial u}$ is irreducible almost everywhere along all solutions of (4.2h). Moreover, by (4.9), we have $A_{i j}(t) \geq 0$ for every $1 \leq i \neq j \leq 4$. It then follows that $w(t)>0$ for all $t>0$ (see, e.g., 47, Proposition 3.1.1 and Remark 3.1.3]).

Without loss of generality, one may even assume that $A_{i j}(t) \geq 0$ for every $1 \leq i, j \leq 4$ and $t>0$ (otherwise, consider $\tilde{A}=A+\mu I$ for some $\mu>0$ ).

Now we claim that $w_{i}(t)>0$ (the subscript $i$ denotes the $i$-th component) for any $t>0$ and $1 \leq i \leq 4$, which means (4.6) is strongly monotone. Otherwise, the index set $K_{1}=\left\{i \in\{1,2,3,4\}: w_{i}\left(t_{i}\right)=0\right.$ for some $\left.t_{i}>0\right\}$ is nonempty. Note also that $w_{i}\left(t_{i}\right)=0$ implies $w_{i}(t)=0$ for any $t \in\left[0, t_{i}\right]$, because $w_{i}(0) \geq 0$ and $\dot{w}_{i}(t) \geq 0$ for all $t>0$. So, we can rewrite $K_{1}=\left\{i \in\{1,2,3,4\}: w_{i}(t)=0, \forall t \in\right.$ $[0, \tilde{t}]$ for some $\tilde{t}>0\}$. Let $K_{2}=\left\{j \in\{1,2,3,4\}: w_{j}(t)>0\right.$ for all $\left.t>0\right\}$. Then $K_{1} \cap K_{2}=\emptyset$ and $K_{1} \cup K_{2}=\{1,2,3,4\}$. Since $w(0)>0$, one has $K_{2} \neq \emptyset$. Moreover, noticing that $A(t)$ is irreducible almost everywhere in $t>0$, one can always choose some $t_{0} \in(0, \tilde{t})$ (no matter how small $\tilde{t}$ is). For such $t_{0}$, there exist an $i_{0} \in K_{1}$ and a $j_{0} \in K_{2}$ such that $A\left(t_{0}\right)_{i_{0} j_{0}}>0$. It then follows that

$$
\dot{w}_{i_{0}}\left(t_{0}\right)=\sum_{l} A\left(t_{0}\right)_{i_{0} l} w_{l}\left(t_{0}\right)=\sum_{l \in K_{2}} A\left(t_{0}\right)_{i_{0} l} w_{l}\left(t_{0}\right) \geq A\left(t_{0}\right)_{i_{0} j_{0}} w_{j_{0}}\left(t_{0}\right)>0 .
$$

On the other hand, the fact that $i_{0} \in K_{1}$ and $t_{0} \in(0, \tilde{t})$ implies that $\dot{w}_{i_{0}}\left(t_{0}\right)=0$, which is a contradiction. We have completed the proof.

Lemma 4.8. If $\sigma_{3}+\sigma_{5} \neq 0$ and $\sigma_{4}+\sigma_{6} \neq 0$, then the Jacobian matrix $\frac{\partial g}{\partial u}(t, u)$ is irreducible almost everywhere along all solutions, and hence $\Pi$ is strongly monotone.

Proof. The last statement is due to Lemma 4.7 directly. We only prove the first statement. Fix $g \in H\left(F_{\sigma}\right)$. By virtue of (4.9) and the uniform-positivity of $\kappa_{i}^{g}(t)$, it is sufficient to prove that $\sigma_{3}+\left(u_{2}-u_{1}\right) \not \equiv 0$ and $\sigma_{4}+\left(u_{4}-u_{3}\right) \not \equiv 0$ in any interval of time $t$. We shall prove that this is the case when $\sigma_{3}+\sigma_{5} \neq 0$ and $\sigma_{4}+\sigma_{6} \neq 0$. Suppose that $\sigma_{4}+\left(u_{4}-u_{3}\right) \equiv 0$ in a time-interval $\left[t_{1}, t_{2}\right]$ along one solution of (4.2, $)$. Then, by equations (4.2,

$$
\begin{aligned}
\kappa_{4}^{g}(t)\left(\sigma_{6}+u_{3}-u_{4}\right) & =\dot{u}_{4}=\dot{u}_{3} \\
& =\kappa_{3}^{g}(t)\left(\sigma_{4}+u_{4}-u_{3}\right)\left(\sigma_{2}+u_{2}-u_{3}\right)-\kappa_{-3}^{g}(t)\left(\sigma_{6}+u_{3}-u_{4}\right),
\end{aligned}
$$

for all $t \in\left[t_{1}, t_{2}\right]$. It then follows that

$$
\left(\kappa_{-3}^{g}(t)+\kappa_{4}^{g}(t)\right)\left(\sigma_{6}+\sigma_{4}\right) \equiv 0, \quad \text { for all } t \in\left[t_{1}, t_{2}\right] .
$$

This contradicts $\sigma_{4}+\sigma_{6} \neq 0$. Similarly, one can also prove that $\sigma_{3}+\left(u_{2}-u_{1}\right) \not \equiv 0$ in any time-interval because $\sigma_{3}+\sigma_{5} \neq 0$. The proof has been completed.

Now we are ready to show the global convergence of the solutions for the timedependent enzymatic futile cycle in mass-action kinetics:

Theorem 4.9. Let $U(t)$ be a solution, with any initial value $U_{0} \in \mathbb{R}_{+}^{6}$, of the time almost-periodic (almost-automorphic) enzymatic-futile-cycle model (4.8). Then 
$U(t)$ is asymptotic to an almost-periodic (almost-automorphic) solution $U^{*}(t)$ of (4.8) with its module $\mathcal{M}\left(U^{*}\right) \subseteq \mathcal{M}(f)$.

In particular, if $\kappa_{i}(t), i= \pm 1,2, \pm 3,4$, in (4.8) are periodic with a common period $T$, then each solution $U(t)$ is asymptotic to a $T$-periodic solution of (4.8). Moreover, if $\kappa_{i}(t), i= \pm 1,2, \pm 3,4$, exhibit different, noncommensurate periods, then each solution $U(t)$ is convergent to a quasi-periodic solution of (4.8).

Proof. For (4.8), note that

$$
U(t)=\left(P(t), Q(t), E_{+}(t), E_{-}(t), C_{+}(t), C_{-}(t)\right)^{T}
$$

for $t \geq 0$. Then $U_{0}=\left(P(0), Q(0), E_{+}(0), E_{-}(0), C_{+}(0), C_{-}(0)\right)^{T}$. For such $U_{0}$, one of the following alternatives holds:

(i): $E_{+}(0)+C_{+}(0)>0$ and $E_{-}(0)+C_{-}(0)>0$; or otherwise

(ii): either $E_{+}(0)+C_{+}(0)$ or $E_{-}(0)+C_{-}(0)$ equals 0 .

We consider these two alternatives separately:

Case (i). Set $\sigma=U_{0} \in \mathbb{R}_{+}^{6}$; one has $\sigma_{3}+\sigma_{5}>0$ and $\sigma_{4}+\sigma_{6}>0$. Then it follows from Lemma 4.8 that $\Pi$ is strongly monotone. Accordingly one can deduce the conclusion directly from Theorem 4.6.

Case (ii). Without loss of generality, we assume that $E_{-}(0)+C_{-}(0)=0$. Since $E_{-}(t)+C_{-}(t)$ satisfies the conservation law, i.e., $E_{-}(t)+C_{-}(t) \equiv$ constant for all $t \in \mathbb{R}$, one obtains that $E_{-}(t)+C_{-}(t) \equiv 0, \forall t \in \mathbb{R}$. Hence $E_{-}(t)=C_{-}(t) \equiv$ $0, \forall t \in \mathbb{R}$, because $U(t)$ is nonnegative. Thus, $\dot{P}=\kappa_{2}(t) C_{+}$in (4.8), which implies that $P(t)$ is nondecreasing. Since $U(t)$ is bounded, $P(t)$ will converge as $t \rightarrow \infty$. Again, noticing that $S(t)+P(t)+C_{+}(t)+C_{-}(t)$ satisfies the conversation law, we have that $S(t)+C_{+}(t)$ will converge decreasingly as $t \rightarrow \infty$. Together with $-\kappa_{2}(t) C_{+}=\frac{d}{d t}\left[S(t)+C_{+}(t)\right] \leq 0$ in (4.8) and the uniform-positivity of $\kappa_{i}$, it then follows that $C_{+}(t)$ will converge as $t \rightarrow \infty$. Consequently, $S(t)$ will also converge as $t \rightarrow \infty$. Of course, $E_{+}(t)$ will converge as well, because $E_{+}(t)+C_{+}(t)$ satisfies a conservation law. Thus, we have proved that all the components of $U(t)$ will converge at $t \rightarrow \infty$ in Case (ii), which completes the proof of the theorem.

Remark 4.10. Theorem 4.9 indicates that, in Case (ii), the chemical reaction will run only in one direction and there will be no cycle. For instance, if the reverse enzymes $\left(E_{-}\right)$and reverse intermediate complex $\left(C_{-}\right)$disappear, then the reaction will only result in the production of $P$. Theorem 4.9 also implies that Case (i) guarantees the occurrence and dynamics of the enzymatic futile cycle.

\section{Application to ReACtion-Diffusion Systems}

Consider the following reaction-diffusion system for an unknown vector-valued function $u(t, x) \in \mathbb{R}^{n}$ on a bounded domain $\Omega \subset \mathbb{R}^{N}$ with smooth boundary $\partial \Omega$ :

$$
\left\{\begin{array}{lr}
u_{t}=D(t) \Delta u+F(t, u), & t>0, \\
\partial u / \partial n=0, & x \in \partial \Omega .
\end{array}\right.
$$

Here $n$ and $N$ are positive integers. $D(t)=\operatorname{diag}\left(d_{1}(t), \cdots, d_{n}(t)\right)$ is a diagonal matrix with all entries greater than some positive constant. The functions $F(t, \cdot)$ : $\mathbb{R}^{n} \rightarrow \mathbb{R}^{n}, D$ and $F$ are sufficiently smooth and $F=\left(f_{1}, \cdots, f_{n}\right)$ satisfies the strong 
cooperativity condition: $\partial f_{i} / \partial u_{j} \geq \delta>0$ for some $\delta>0$ and all $i, j=1, \cdots, n$ with $i \neq j$. We also assume that both $D$ and $F$ are admissible and time almostperiodic (almost-automorphic). Of course, here $\Delta$ is the Laplacian and $\partial / \partial n$ is the unit outward normal vector field on $\partial \Omega$.

Let $v^{*}=\left(v_{1}^{*}, \cdots, v_{n}^{*}\right) \in \operatorname{Int} \mathbb{R}_{+}^{n}$, with all the components $v_{i}^{*}, 1 \leq i \leq n$, being a fixed positive vector. We assume that the nonlinearity $F$ satisfies

$$
F\left(t, u+a v^{*}\right)=F(t, u), \quad \text { for any } a \in \mathbb{R}, u \in \mathbb{R}^{n} \text { and } t \geq 0 .
$$

We further make the following additional assumption: For each $v_{0} \in \mathbb{R}^{n}$, the corresponding ODE

$$
\frac{d v}{d t}=h(t, v), \quad v(0)=v_{0}
$$

possesses a solution bounded on $[0,+\infty)$ uniformly for all $h$ in the hull $H(F)$.

Let $Y$ be the hull $H(D, F)$ and $X=\left\{u \in C\left(\bar{\Omega}, \mathbb{R}^{n}\right): \partial u /\left.\partial n\right|_{\partial \Omega}=0\right\}$. Then one can define a skew-product semiflow $\Pi$ on $X \times Y$ by the solutions of (5.1) (cf. [17, Sec. 3.4], [32] or [6, Sec. 6]). Strong cooperativity in conjunction with the strong comparison principle implies the strong monotonicity of $\Pi$ on $X \times Y$ (cf. [40]). Also, it follows from the work in [17] and the standard a priori estimates for parabolic equations that $\Pi$ is completely continuous. Let $G$ be the phase-translation group with respect to $v^{*} \in \operatorname{Int} \mathbb{R}_{+}^{n}$. Then it is not difficult to check that $G$ commutes with $\Pi$.

Applying our main results in Section 3, we obtain a convergence result for reaction-diffusion system (5.1):

Theorem 5.1. Any solution $u(t)$ of (5.1) will be asymptotic to an almost-periodic (almost-automorphic) solution $u^{*}(t)$ of (5.1) with its module $\mathcal{M}\left(u^{*}\right) \subseteq \mathcal{M}(F)$. In particular, $u(t)$ is asymptotic to a T-periodic solution when the nonlinearity $F$ is T-periodic in time $t$.

\section{ACKNOWLEDGEMENT}

The authors are indebted to an anonymous referee for valuable comments and suggestions which led to the improvement of an earlier version of this paper.

\section{REFERENCES}

[1] N.D. Alikakos and P. Hess, On stabilization of discrete monotone dynamical systems, Israel J. Math. 59 (1987), 185-194. MR.920081 (89h:47083)

[2] N.D. Alikakos, P. Hess and H. Matano, Discrete order preserving semigroups and stability for periodic parabolic differential equations, J. Diff. Equ. 82 (1989), 322-341. MR 1027972 (91i:35016)

[3] D. Angeli, P. Leenheer and E.D. Sontag, Graph-theretic characterizations of monotonicity of chemical networks in reaction coordinates, J. Math. Biol. 61 (2010), 581-616. MR2672536

[4] D. Angeli and E.D. Sontag, Translation-invariant monotone systems, and a global convergence result for enzymatic futile cycles, Nonlinear Anal. Ser B: Real World Appl. 9 (2008), 128-140. MR2370168 (2008k:92019)

[5] O. Arino, Monotone semi-flows which have a monotone first integral, in: Delay Differential Equations and Dynamical Systems, Springer Lect. Notes Math. 1475 (1991), 64-75. MR:1132019 (93a:34081) 
[6] A. V. Babin and G. Sell, Attractors of non-autonomous parabolic equations and their symmetry properties, J. Diff. Equ. 160 (2000), 1-50. MR.1734528(2001i:35032)

[7] J.J. Bijlsma and E.A. Groisman, Making informed decisions: Regulatory interactions between two-component systems, Trends Microbiol. 11 (2003), 359-366.

[8] R.G. Casten and C.J. Holland, Instability results for reaction-diffusion equations with Neumann boundary conditions, J. Diff. Equ. 27 (1978), 266-273. MR 480282 (80a:35064)

[9] K. Cho, S. Shin, H. Kim, O.Wolkenhauer, B. McFerran and W. Kolch, Mathematical modeling of the influence of RKIP on the ERK signaling pathway, Computational Methods in Systems Biology, CMSB03, Lecture Notes in Computer Science, vol. 2602, Springer, Berlin, 2003.

[10] K. Deimling, Nonlinear Functional Analysis, Springer-Verlag, Berlin Heidelberg, 1985. MR787404 (86j:47001)

[11] S. Donovan, K.M. Shannon and G. Bollag, GTPase activating proteins: Critical regulators of intracellular signaling, Biochim, Biophys. Acta 1602 (2002), 23-45.

[12] M. Feinberg, Some recent results in chemical reaction network theory. In: R. Aris, D. G. Aronson, and H. L. Swinney (eds.) Patterns and dynamics in reactive media, IMA Vol. Math. Appl. 37, Springer, Berlin, 1991, 43-170. MR1228917 (94b:80009)

[13] J.E. Ferrell, Jr., Tripping the switch fantastic: How a protein kinase cascade can convert graded inputs into switch-like outputs, Trends Biochem. Sci. 21 (1996), 460-466.

[14] A. M. Fink, Almost periodic differential equations, Lect. Notes Math. 840 (Springer-Verlag, Berlin, 1981).

[15] A.D. Grossman, Genetic networks controlling the intiation of sporulation and the development of genetic competence in Bacillus subtilis, Annu. Rev. Genet. 29 (1995), 477-508.

[16] J.R. Haddock, M.N. Nkashama and J. Wu, Asymptotic constancy for pseudo monotone dynamical systems on function spaces, J. Diff. Equ. 100 (1992), 292-311. MR1194812 (94b:34096)

[17] D. Henry, Geometric Theory of Semilinear Parabolic Equations, Lecture Notes in Mathematics, Vol. 840, Springer, New York, 1981. MR610244 (83j:35084)

[18] M. Hirsch, The dynamical systems approach to differential equations, Bull. Amer. Math. Soc. 11 (1984), 1-64. MR741723 (85m:58060)

[19] M. Hirsch, Stability and convergence in strongly monotone dynamical systems, J. Reine Angew. Math. 383 (1988), 1-53. MR921986 (89c:58108)

[20] M. Hirsch and H. Smith, Monotone dynamical systems. in: A. Canada, P. Drabek, A. Fonda (Eds.), Handbook of Differential Equations, Ordinary Differential Equations, second volume, Elsevier, Amsterdam, 2005. MR2182759 (2006j:37017)

[21] H. Hu and J. Jiang, Translation-invariant monotone systems, I: Autonomous/periodic case, Nonlinear Analysis: RWA 11 (2010), 3211-3217. MR2661981

[22] H. $\mathrm{Hu}$ and J. Jiang, Translation-invariant monotone systems, II: Almost periodic/automorphic case, Proc. Amer. Math. Soc. 138 (2010), 3997-4007. MR2679621

[23] J. Jiang, Sublinear discrete-time order-perserving dynamical systems, Math. Proc. Camb. Phil. Soc. 119 (1996), 561-574. MR.1357065 (96h:34090)

[24] J. Jiang, Periodic monotone systems with an invariant function, SIAM J. Math. Anal. 27 (1996), 1738-1744. MR:1416516 (98h:34089)

[25] J. Jiang and X.-Q. Zhao, Convergence in monotone and uniformly stable skew-product semiflows with applications, J. Reine Angew. Math. 589 (2005), 21-55. MR2194677(2006k:37031)

[26] J. Keener and J. Sneyd, Mathematical Physiology, Springer, New York, 1998. MR.1673204 (2000c:92010)

[27] B.N. Kholodenko, A. Kiyatkin, F. Bruggenman, E.D. Sontag, H. Westerhoff and J. Hoek, Untangling the wires: A novel strategy to trace gunctional interactions in signaling and gene networks, Proc. Nat. Acad. Sci. USA 99 (2002), 12841-12846.

[28] D.J. Lew and D.J. Burke, The spindle assembly and spindle position checkpoings, Annu. Rev. Genet. 37 (2003), 251-282.

[29] H. Matano, Asymptotic behavior and stability of solutions of semilinear diffusion equations, Publ. RIMS, Kyoto Univ. 15 (1979), 401-454. MR555661 (80m:35046)

[30] J. Mierczyński, Strictly cooperative systems with a first integral, SIAM J. Math. Anal. 18 (1987), 642-646. MR883558 (88e:34093)

[31] J. Mierczyński and P. Poláčik, Group actions on strongly monotone dynamical systems, Math. Ann. 283 (1989), 1-11. MR973801 (90k:58182) 
[32] J. Mierczynski and W. Shen, Spectral Theory for Random and Nonautonomous Parabolic Equations and Applications, Chapman Hall/CRC Monographs and Surveys in Pure and Applied Mathematics, 139, Chapman, 2008. MR2464792 (2010g:35216)

[33] S. Novo and R. Obaya, Strictly ordered minimal subsets of a class of convex monotone skewproduct semiflows, J. Diff. Equ. 196 (2004), 249-288. MR.2025194(2004i:37032)

[34] S. Novo, R. Obaya and A. Sanz, Stability and extensibility results for abstract skew-product semiflows, J. Diff. Equ. 235 (2007), 623-646. MR2317498 (2008h:37018)

[35] T. Ogiwara and H. Matano, Stability analysis in order-preserving systems in the presence of symmetry, Proc. R. Soc. Edinburgh 129 (1999), 395-438. MR.1686708(2000m:37175)

[36] T. Ogiwara and H. Matano, Monotonicity and convergence results in order-preserving systems in the presence of symmetry, Discrete Contin. Dyn. Syst. 5 (1999), 1-34. MR.1664441 (2000k:37126)

[37] H.G. Othmer, Analysis of complex reaction networks, (2003) Available on-line at http://www.math. leidenuniv.nl/ verduyn.

[38] P. Poláčik, Convergence in smooth strongly monotone flows defined by semilinear parabolic equation, J. Diff. Equ. 79 (1989), 89-110. MR997611 (90f:58025)

[39] P. Poláčik and I. Tereščák, Convergence to cycles as a typical asymptotic behavior in smooth strongly monotone discrete-time dynamical systems, Arch. Rat. Mech. Anal. 116 (1991), 339-360. MR1132766 (93b:58088)

[40] H. Protter and H. Weinberger, Maximum Principles in Differential Equations, Prentice-Hall, Englewood Cliffs, N.J., 1967. MR0219861 (36:2935)

[41] M. Samoilov, S. Plyasunov and A.P. Arkin, Stochastic amplification and signaling in enzymatic futile cycles through noise-induced bistability with oscillations, Proc. Nat. Acad. Sci. USA 102 (2005), 2310-2315.

[42] G.R. Sell, Nonautonomous differential equations and topological dynamics, Trans. Amer Math. Soc. 127 (1967), 241-283. MR0212313 (35:3187a)

[43] G.R. Sell, Topological Dynamics and Ordinary Differential Equations, Von Norstand Reinhold Company, London, 1971. MR0442908 (56:1283)

[44] W. Shen and Y. Yi, Almost automorphic and almost periodic dynamics in skew-product semiflows, Mem. Amer. Math. Soc. no. 647, 136, Providence, R.I. (1998). MR1445493(99d:34088)

[45] W. Shen and X.-Q. Zhao, Convergence in almost periodic cooperative systems with a first integral, Proc. Amer. Math. Soc. 133 (2005), 203-212. MR2085171 (2005d:34076)

[46] H.L. Smith, Cooperative systems of differential equations with concave nonlinearities, Nonlin. Anal. TMA 10 (1986), 1037-1052. MR857738 (87k:58247)

[47] H.L. Smith, Monotone Dynamical Systems, An Introduction to the Theory of Competitive and Cooperative Systems, Math. Surv. Monogr. 41, Amer. Math. Soc., Providence, RI, 1995. MR1319817 (96c:34002)

[48] E.D. Sontag, Some new directions in control theory inspired by systems biology, Systems Biol. 1 (2004), 9-18.

[49] L. Stryer, Biochemistry, Freeman, New York, 1995.

[50] M.L. Sulis and R. Parsons, PTEN: from pathology to biology, Trends Cell Biol. 13 (2003), $478-483$.

[51] P. Takáč, Asymptotic behavior of strongly monotone time-periodic dynamical process with symmetry, J. Diff. Equ. 100 (1992), 355-378. MR1194815 (94d:47060)

[52] P. Takáč, Asymptotic behavior of discrete-time semigroups of sublinear, strongly increasing mappings with applications in biology, Nonlin. Anal. TMA 14 (1990), 35-42. MR.1028245 (90j:47088)

[53] B. Tang, Y. Kuang and H. Smith, Strictly nonautonomous cooperative system with a first integral, SIAM J. Math. Anal. 24 (1993), 1331-1339. MR.1234019 (94g:34067)

[54] W. A. Veech, Almost automorphic functions on groups, Amer. J. Math. 87 (1965), 719-751. MR0187014 (32:4469)

[55] Y. Wang, Asymptotic symmetry in strongly monotone skew-product semiflows with applications, Nonlinearity 22 (2009), 765-782. MR2486356 (2010j:35247)

[56] Y. Wang and X.-Q. Zhao, Convergence in monotone and subhomogeneous discrete dynamical systems on product Banach space, Bull. London Math. Soc. 35 (2003), 681-688. MR.1989498 (2004c:35157)

[57] J. Wu, Convergence of monotone dynamical systems with minimal equilibria, Proc. Amer. Math. Soc. 106 (1989), 907-911. MR1004632 (90j:58130) 
[58] X.-Q. Zhao, Global attractivity in monotone and subhomogeneous almost periodic systems, J. Diff. Equ. 187 (2003), 494-509. MR.1949451(2004i:37157)

Department of Mathematics, University of Science and Technology of China, Hefei, Anhui, 230026, People's Republic of China

Wu Wen-Tsun Key Laboratory of Mathematics, Department of Mathematics, University of Science and Technology of China Hefei, Anhui, 230026, People's Republic of China - and - Department of Mathematics and Statistics, University of Helsinki, P.O. Box 68, FIN-00014, Finland 